\title{
Existence and Stability Results for Semilinear Systems of Impulsive Stochastic Differential Equations with Fractional Brownian Motion
}

\author{
T. Blouhi ${ }^{1}$, T. Caraballo ${ }^{2}$ and A. Ouahab ${ }^{1}$ \\ ${ }^{1}$ Laboratory of Mathematics, Univ Sidi Bel Abbes \\ PoBox 89, 22000 Sidi-Bel-Abbès, Algeria. \\ E-mail: blouhitayeb@yahoo.com, agh_ouahab@yahoo.fr \\ 2 Depto. Ecuaciones Diferenciales y Análisis Numérico, \\ Universidad de Sevilla, Campus de Reina Mercedes \\ 41012-Sevilla, Spain \\ E-mail: caraball@us.es
}

\begin{abstract}
Some results on the existence and uniqueness of mild solution for a system of semilinear impulsive differential equations with infinite fractional Brownian motions are proved. The approach is based on Perov's fixed point theorem and a new version of Schaefer's fixed point theorem in generalized Banach spaces. The relationship between mild and weak solutions and the exponential stability of mild solutions are investigated as well. The abstract theory is illustrated with an example.
\end{abstract}

Key words and phrases: Mild solutions, fractional Brownian motion, impulsive differential equations, matrix convergent to zero, generalized Banach space, fixed point. AMS (MOS) Subject Classifications: 34A37,60H99,47H10.

\section{Introduction}

Differential equations with impulses were considered for the first time by Milman and Myshkis [23] and then it was followed by a period of active research which culminated with the monograph by Halanay and Wexler [15]. Many phenomena and evolution processes in physics, chemical technology, population dynamics, and natural sciences may change their state abruptly or be subject to short-term perturbations. These perturbations may be seen as impulses. Impulsive problems arise also in various applications in communications, mechanics (jump discontinuities in velocity), electrical engineering, medicine and biology. A comprehensive introduction to the basic theory is well developed in the monographs by Benchohra et al. [2], Graef et al. [14], Laskshmikantham et al. [17], Samoilenko and Perestyuk [35].

Random differential and integral equations play an important role in characterizing many social, physical, biological and engineering problems; see for instance the 
monographs by Da Prato and Zabczyk [11], Gard [12], Gikhman and Skorokhod [13], Sobzyk [36] and Tsokos and Padgett [37]. For example, a stochastic model for drug distribution in a biological system was described by Tsokos and Padgett [37] as a closed system with a simplified heart, one organ or capillary bed, and re-circulation of a blood with a constant rate of flow, where the heart is considered as a mixing chamber of constant volume. For the basic theory concerning stochastic differential equations see the monographs by Bharucha-Reid [3], Mao [22], Øksendal, [26], Tsokos and Padgett [37], Sobczyk [36] and Da Prato and Zabczyk [11].

The study of impulsive stochastic differential equations is a relatively new research area. The existence and stability of stochastic impulsive differential equations were recently investigated, for example in [8,14, 18-20, 27, 34, 38, 39].

This paper is concerned with a system of stochastic impulsive differential equations of the following type:

$$
\left\{\begin{aligned}
d x(t) & =\left(A x(t)+f^{1}(t, x(t), y(t)) d t\right. \\
& \left.+\sum_{l=1}^{\infty} \sigma_{l}^{1}(t, x(t)), y(t)\right) d B_{l}^{H}(t), t \in[0, T], t \neq t_{k}, \\
d y(t) & =\left(A y(t)+f^{2}(t, x(t), y(t))\right) d t \\
& +\sum_{l=1}^{\infty} \sigma_{l}^{2}(t, x(t), y(t)) d B_{l}^{H}(t), t \in J, t \neq t_{k}, \\
\Delta x(t) & =I_{k}\left(x\left(t_{k}\right)\right), \quad t=t_{k} \quad k=1,2, \ldots, m \\
\Delta y(t) & =\bar{I}_{k}\left(y\left(t_{k}\right)\right), \quad t=t_{k} \quad k=1,2, \ldots, m \\
x(0) & =x_{0} \\
y(t) & =y_{0}
\end{aligned}\right.
$$

where $X$ is a real separable Hilbert space with inner product $\langle\cdot, \cdot\rangle$ and induced norm $\|\cdot\|$, $A: D(A) \subset X \longrightarrow X$ is the infinitesimal generator of a strongly continuous semigroup of bounded linear operators $(S(t))_{t \geq 0}$ in $X$ and $f^{1}, f^{2}:[0, T] \times X \times X \longrightarrow X$ are given functions, $B_{l}^{H}$ is an infinite sequence of mutually independent fractional Brownian motions, $l=1,2, \ldots$, with Hurst parameter $H, I_{k}, \bar{I}_{k} \in C(X, X)(k=1,2, \ldots, m)$, $\sigma_{l}^{1}, \sigma_{l}^{2}: J \times X \times X \rightarrow L_{Q}^{0}(Y, X)$. Here, $L_{Q}^{0}(Y, X)$ denotes the space of all $Q$-HilbertSchmidt operators from $Y$ into $X$, which will be also defined in the next section. Moreover, the fixed times $t_{k}$ satisfy $0<t_{1}<t_{2}<\ldots<t_{m}<T$, and $y\left(t_{k}^{-}\right)$and $y\left(t_{k}^{+}\right)$ denote the left and right limits of $y(t)$ at $t=t_{k}$.

$$
\left\{\begin{array}{l}
\sigma(t, x, y)=\left(\sigma_{1}(t, x, y), \sigma_{2}(t, x, y), \ldots\right) \\
\|\sigma(t, x, y)\|^{2}=\sum_{j=1}^{\infty}\left\|\sigma_{j}(t, x, y)\right\|_{L_{Q}^{0}}^{2}<\infty
\end{array}\right.
$$

where $\sigma(\cdot, \cdot, \cdot) \in \ell^{2}$, and $\ell^{2}$ is given by

$$
\ell^{2}=\left\{\phi=\left(\phi_{j}\right)_{j \geq 1}:[0, T] \times X \times X \rightarrow L_{Q}^{0}(Y, X) \quad:\|\phi(t, x, y)\|^{2}=\sum_{j=1}^{\infty}\left\|\phi_{j}(t, x, y)\right\|_{L_{Q}^{0}}^{2}<\infty\right\} .
$$


We denote $a \wedge b=\min (a, b)$ and $a \vee b=\max (a, b)$. It is obvious that system (1.1) can be seen as a fixed point problem for the model

$$
\left\{\begin{array}{l}
\left.d z(t)=A_{*} z(t)+f(t, z(t)) d t+\sum_{l=1}^{\infty} \sigma_{l}(t, z)\right) d B_{l}^{H}(t), t \in[0, T], t \neq t_{k}, \\
\Delta z(t)=I_{k}^{*}\left(z\left(t_{k}\right)\right), \quad t=t_{k} \quad k=1,2, \ldots, m \\
z(0)=z_{0}
\end{array}\right.
$$

where

$z(t)=\left[\begin{array}{l}x(t) \\ y(t)\end{array}\right], A_{*}=\left[\begin{array}{cc}A & 0 \\ 0 & A\end{array}\right], f(t, z)=\left[\begin{array}{l}f^{1}(t, x(t), y(t)) \\ f^{2}(t, x(t), y(t))\end{array}\right], \sigma_{l}(t, z)=\left[\begin{array}{c}\sigma_{l}^{1}(t, x, y) \\ \sigma_{l}^{2}(t, x, y)\end{array}\right]$ and $z_{0}=\left[\begin{array}{l}x_{0} \\ y_{0}\end{array}\right]$.

In the deterministic framework, the above system was used to study initial value problems and boundary value problems for nonlinear competitive or cooperative differential systems from mathematical biology [24] and mathematical economics [16] where the model is usually considered in the operator form (1.1).

Some results on the existence of solutions for differential equations with infinite Brownian motions were obtained in $[7,38]$. Existence and uniqueness of mild solutions to neutral stochastic delay functional integro-differential equations perturbed by a fractional Brownian motion can be found in Caraballo and Diop [10].

Very recently, for $A \equiv 0, X=\mathbb{R}^{n}$ and $B_{l}^{H}$ a standard Brownian motion, problem (1.1) was studied by Blouhi et. al. [4]. In $[5,6,31,32]$ the authors present existence and uniqueness results for systems of semilinear differential equations without impulses. Recently, Precup [31] proved the role of matrix convergence and vector metrics in the analysis of semilinear operator systems.

The aim of this paper is to study existence, uniqueness and exponential stability of mild solutions of semilinear systems of stochastic differential equations with infinite fractional Brownian motions and impulses. The paper is organized as follows. In sections 2 and 3 we introduce all the background material used in this paper such as stochastic calculus and some properties of generalized Banach spaces. In Section 4 we state and prove our main results by using Perov's and Schaefer's fixed point theorems in generalized Banach spaces. Finally, sections 5 and 6 are devoted to prove the relationship between mild and weak solutions, and exponential stability of solutions for Problem (1.1). Some application examples are finally considered in the last section.

\section{Preliminaries}

In this section, we introduce notations, definitions, and preliminary facts which are used throughout this paper. 
Let $(\Omega, \mathcal{F}, \mathbb{P})$ be a complete probability space with a filtration $\left(\mathcal{F}=\mathcal{F}_{t}\right)_{t \geq 0}$ satisfying the usual conditions (i.e. right continuous and $\mathcal{F}_{0}$ containing all $\mathbb{P}$-null sets).

For a stochastic process $x(\cdot, \cdot):[0, T] \times \Omega \rightarrow X$ we will write $x(t)$ (or simply $x$ when no confusion is possible) instead of $x(t, \omega)$.

Definition 2.1. Given $H \in(0,1)$, a continuous centered Gaussian process $B^{H}$ is said to be a two-sided one-dimensional fractional Brownian motion ( $\mathrm{fBm}$ ) with Hurst parameter $H$, if its covariance function $\left.R_{H}(t, s)=E\left[B^{H}(t)\right) B^{H}(s)\right]$ satisfies

$$
R_{H}(t, s)=\frac{1}{2}\left(|t|^{2 H}+|s|^{2 H}-|t-s|^{2 H}\right) \quad t, s \in[0, T] .
$$

It is known that $B^{H}(t)$ with $H>\frac{1}{2}$ admits the following Volterra representation

$$
B^{H}(t)=\int_{0}^{t} K_{H}(t, s) d B(s)
$$

where $B$ is a standard Brownian motion given by

$$
B(t)=B^{H}\left(\left(K_{H}^{*}\right)^{-1} \xi_{[0, t]}\right),
$$

and the Volterra kernel the kernel $K(t, s)$ is given by

$$
K_{H}(t, s)=c_{H} s^{1 / 2-H} \int_{s}^{t}(u-s)^{H-\frac{3}{2}}\left(\frac{u}{s}\right)^{H-\frac{1}{2}} d u, \quad t \geq s,
$$

where $c_{H}=\sqrt{\frac{H(2 H-1)}{\beta\left(2 H-2, H-\frac{1}{2}\right)}}$ and $\beta(\cdot, \cdot)$ denotes the Beta function, $K(t, s)=0$ if $t \leq s$, and it holds

$$
\frac{\partial K_{H}}{\partial t}(t, s)=c_{H}\left(\frac{t}{s}\right)^{H-\frac{1}{2}}(t-s)^{H-\frac{3}{2}},
$$

and the kernel $K_{H}^{*}$ is defined as follows. Denote by $\mathcal{E}$ the set of step functions on $[0, T]$. Let $\mathcal{H}$ be the Hilbert space defined as the closure of $\mathcal{E}$ with respect to the scalar product

$$
\left\langle\chi_{[0, t]}, \chi_{[0, s]}\right\rangle_{\mathcal{H}}=R_{H}(t, s),
$$

and consider the linear operator $K_{H}^{*}$ from $\mathcal{E}$ to $L^{2}([0, T])$ defined by,

$$
\left(K_{H}^{*} \phi\right)(t)=\int_{s}^{T} \phi(t) \frac{\partial K_{H}}{\partial t}(t, s) d t
$$

Notice that,

$$
\left(K_{H}^{*} \chi_{[0, t]}\right)(s)=K_{H}(t, s) \chi_{[0, t]}(s) .
$$

The operator $K_{H}^{*}$ is an isometry between $\mathcal{E}$ and $L^{2}([0, T])$ which can be extended to the Hilbert space $\mathcal{H}$. In fact, for any $s, t \in[0, T]$ we have

$$
\left\langle K_{H}^{*} \chi_{[0, t]}, K_{H}^{*} \chi_{[0, t]}\right\rangle_{L^{2}([0, T])}=\left\langle\chi_{[0, t]}, \chi_{[0, s]}\right\rangle_{\mathcal{H}}=R_{H}(t, s) .
$$


In addition, for any $\phi \in \mathcal{H}$,

$$
\int_{0}^{T} \phi(s) d B^{H}(s)=\int_{0}^{T}\left(K_{H}^{*} \phi\right)(s) d B(s)
$$

if and only if $K_{H}^{*} \phi \in L^{2}([0, T])$. Moreover, the following useful result holds

Lemma 2.1. [25] There exists a positive constant $c_{1}(H)$ such that for any $\phi \in$ $L^{1 / H}([0, T])$ it holds

$$
H(2 H-1) \int_{0}^{T} \int_{0}^{T}|\phi(y)\|\phi(z)\| y-z|^{2 H-2} d y d z \leq c_{1}(H)\|\phi\|_{L^{1 / H}([0, T])}^{2} .
$$

Next we are interested in considering an $\mathrm{fBm}$ with values in a Hilbert space and giving the definition of the corresponding stochastic integral.

Definition 2.2. An $\mathcal{F}_{t}$-adapted process $\phi$ on $[0, T] \times \Omega \rightarrow X$ is an elementary or simple process if for a partition $\psi=\left\{\bar{t}_{0}=0<\bar{t}_{1}<\ldots<\bar{t}_{n}=T\right\}$ and $\left(\mathcal{F}_{\bar{t}_{i}}\right)$-measurable $X$ valued random variables $\left(\phi_{\bar{t}_{i}}\right)_{1 \leq i \leq n}, \phi_{t}$ satisfies

$$
\phi_{t}(\omega)=\sum_{i=1}^{n} \phi_{i}(\omega) \chi_{\left(\bar{t}_{i-1}, \bar{t}_{i}\right]}(t), \quad \text { for } \quad 0 \leq t \leq T, \quad \omega \in \Omega .
$$

The Itô integral of the simple process $\phi$ is defined as

$$
I_{H}(\phi)=\int_{0}^{T} \phi_{l}(s) d B_{l}^{H}(s)=\sum_{i=1}^{n} \phi_{l}\left(\bar{t}_{i}\right)\left(B_{l}^{H}\left(\bar{t}_{i}\right)-B_{l}^{H}\left(\bar{t}_{i-1}\right)\right),
$$

whenever $\phi_{\bar{t}_{i}} \in L^{2}\left(\Omega, \mathcal{F}_{\bar{t}_{i}} \mathbb{P}, X\right)$ for all $i \leq n$.

Let $\left(X,\langle\cdot, \cdot\rangle,|\cdot|_{X}\right),\left(Y,\langle\cdot, \cdot\rangle,|\cdot|_{Y}\right)$ be separable Hilbert spaces. Let $\mathcal{L}(Y, X)$ denote the space of all linear bounded operators from $Y$ into $X$. Let $e_{n}, n=1,2, \ldots$ be a complete orthonormal basis in $Y$ and $Q \in \mathcal{L}(Y, X)$ be an operator defined by $Q e_{n}=\lambda_{n} e_{n}$ with finite trace $\operatorname{tr} Q=\sum_{n=1}^{\infty} \lambda_{n}<\infty$ where $\lambda_{n}, n=1,2, \ldots$, are non-negative real numbers. Let $\left(\beta_{n}^{H}\right)_{n \in N}$ be a sequence of two-sided one-dimensional standard fractional Brownian motions mutually independent on $(\Omega, \mathcal{F}, \mathbb{P})$. If we define the infinite dimensional $f B m$ on $Y$ with covariance $Q$ as

$$
B^{H}(t)=\sum_{n=1}^{\infty} \sqrt{\lambda_{n}} \beta_{n}^{H}(t) e_{n}
$$

then it is well defined as an $Y$-valued $Q$-cylindrical fractional Brownian motion (see [9]) and we have

$$
E\left\langle\beta_{l}^{H}(t), x\right\rangle\left\langle\beta_{k}^{H}(s), y\right\rangle=R_{H_{l k}}(t, s)\langle Q(x), y\rangle, \quad x, y \in Y \quad \text { and } s, t \in[0, T]
$$


such that

$$
R_{H_{l k}}=\frac{1}{2}\left\{|t|^{2 H}+|s|^{2 H}+|t-s|^{2 H}\right\} \delta_{l k} \quad t, s \in[0, T]
$$

where

$$
\delta_{l j}= \begin{cases}1 & j=l \\ 0, & j \neq l\end{cases}
$$

In order to define Wiener integrals with respect to a $Q-f B m$, we introduce the space $L_{Q}^{0}:=L_{Q}^{0}(Y, X)$ of all $Q$-Hilbert-Schmidt operators $\varphi: Y \longrightarrow X$. We recall that $\varphi \in L(Y, X)$ is called a $Q$-Hilbert-Schmidt operator, if

$$
\|\varphi\|_{L_{Q}^{0}}^{2}=\left\|\varphi Q^{1 / 2}\right\|_{H S}^{2}=\operatorname{tr}\left(\varphi Q \varphi^{*}\right)<\infty .
$$

Definition 2.3. Let $\phi(s), s \in[0, T]$, be a function with values in $L_{Q}^{0}(Y, X)$. The Wiener integral of $\phi$ with respect to $\mathrm{fBm}$ given by (2.4) is defined by

$$
\begin{gathered}
\int_{0}^{T} \phi(s) d B^{H}(s)=\sum_{n=1}^{\infty} \int_{0}^{t} \sqrt{\lambda_{n}} \phi(s) e_{n} d \beta_{n}^{H} \\
=\sum_{n=1}^{\infty} \int_{0}^{T} \sqrt{\lambda_{n}} K_{H}^{*}\left(\phi e_{n}\right)(s) d \beta_{n} .
\end{gathered}
$$

Notice that if

$$
\sum_{n=1}^{\infty}\left\|\phi Q^{1 / 2} e_{n}\right\|_{L^{1 / H}([0, T] ; X)}<\infty
$$

the next result ensures the convergence of the series in the previous definition. It can be proved by similar arguments to those used to prove Lemma 2 in Caraballo et al. [9] and Lemma 2.1 in Blouhi et al [4].

Lemma 2.2. For any $\phi:[0, T] \rightarrow L_{Q}^{0}(Y, X)$ such that (2.6) holds, and for any $\alpha, \beta \in$ $[0, T]$ with $\alpha>\beta$,

$$
E\left|\int_{\alpha}^{\beta} \phi(s) d B^{H}(s)\right|_{X}^{2} \leq c_{2}(H) H(2 H-1)(\alpha-\beta)^{2 H-1} \sum_{n=1}^{\infty} \int_{\alpha}^{\beta}\left|\phi(s) Q^{1 / 2} e_{n}\right|_{X}^{2} d s .
$$

where $c_{2}(H)$ is a constant depending on $H$. If, in addition,

$$
\sum_{n=1}^{\infty}\left|\phi Q^{1 / 2} e_{n}\right|_{X} \text { is uniformly convergent for } t \in[0, T] \text {, }
$$

then,

$$
E\left|\int_{\alpha}^{\beta} \phi(s) d B^{H}(s)\right|_{X}^{2} \leq c_{2}(H) H(2 H-1)(\alpha-\beta)^{2 H-1} \int_{\alpha}^{\beta}\|\phi(s)\|_{L_{Q}^{0}}^{2} d s .
$$


Corollary 2.1. Let $\left(\mathcal{F}_{t}\right)_{t \geq 0}$ be a right-continuous complete $\sigma$-algebras filtration generated by a sequence of $f B m B_{l}^{H}, l=1,2, \ldots$, mutually independent, and consider another sequence of $\left(\mathcal{F}_{t}\right)$-adapted processes $\phi_{l}$. Denote

$$
M^{k}(t)=\sum_{l=1}^{k} \int_{0}^{t} \phi_{l}(y) d B_{l}^{H}(y), k \in \mathbb{N},
$$

and assume that

$$
\sum_{l=1}^{\infty} \int_{0}^{T} \int_{0}^{T}\left\|\phi_{l}(y)\right\|_{L_{Q}^{0}}\left\|\phi_{l}(z)\right\|_{L_{Q}^{0}}|y-z|^{2 H-2} d y d z<\infty
$$

for all $t \geq 0$. Then, $M^{k}$ is a convergent in $L^{2}\left(\Omega, \mathcal{F}_{t}, \mathbb{P} ; X\right)$

Proof. Let $M^{k}(t)=\left(\sum_{l=1}^{k} \int_{0}^{t} \phi_{l}(y) d B_{l}^{H}(y)\right)$ for any $t \in[0, T]$. By Lemma 2.2, we have that

$$
\begin{aligned}
E\left|M^{n}(t)-M^{m}(t)\right|_{X}^{2} & =E\left|\sum_{l=1}^{n} \int_{0}^{t} \phi_{l}(y) d B_{l}^{H}(y)-\sum_{l=1}^{m} \int_{0}^{t} \phi_{l}(y) d B_{l}^{H}(y)\right|_{X}^{2} \\
& =E\left|\sum_{l=n \wedge m+1}^{n \vee m} \int_{0}^{t} \phi_{l}(y) d B_{l}^{H}(y)\right|_{X}^{2} \sum_{l=n \wedge m+1}^{n \vee m} \int_{0}^{T}\left\|\phi_{l}(y)\right\|_{L_{Q}^{0}}^{2} d y \rightarrow 0 \\
& \leq c_{3}(H) H(2 H-1) T^{2 H-1} \sum^{n}(y)
\end{aligned}
$$

as $n, m \rightarrow \infty$ where $c_{3}(H)>0$. Consequently $M^{n}(\cdot)$ is a Cauchy sequence with respect to the norm $\sup _{0 \leq t \leq T} E|\cdot|_{X}^{2}$ and the limit is $M$. Then we can conclude

$$
\lim _{n \rightarrow \infty} \sup _{0 \leq t \leq T} E\left|M^{n}(t)-M(t)\right|_{X}^{2}=0
$$

and observe that

$$
\sum_{l=1}^{\infty} \int_{0}^{T} \int_{0}^{T}\left\|\phi_{l}(y)\right\|_{L_{Q}^{0}}\left\|\phi_{l}(z)\right\|_{L_{Q}^{0}}|y-z|^{2 H-2} d y d z<\infty .
$$

Then $M$ in $L^{2}\left(\Omega, \mathcal{F}_{t}, \mathbb{P} ; X\right)$.

The following result is one of the elementary properties of square-integrable stochastic processes. 
Lemma 2.3. (Itô Isometry for Elementary Processes) Let $\left(\phi_{l}\right)_{l \in \mathbb{N}}$ be a sequence of elementary processes. If

$$
\sum_{l=1}^{\infty} \int_{0}^{T} \int_{0}^{T}\left\|\phi_{l}(y)\right\|_{L_{Q}^{0}}\left\|\phi_{l}(z)\right\|_{L_{Q}^{0}}|y-z|^{2 H-2} d y d z<\infty
$$

then

$E\left|\sum_{l=1}^{\infty} \int_{0}^{T} \phi_{l}(y) d B_{l}^{H}(y)\right|_{X}^{2} \leq H(2 H-1) \sum_{l=1}^{\infty} \int_{0}^{T} \int_{0}^{T}\left\|\phi_{l}(y)\right\|_{L_{Q}^{0}}\left\|\phi_{l}(z)\right\|_{L_{Q}^{0}}|y-z|^{2 H-2} d y d z$.

Proof. Let $M^{k}=\sum_{l=1}^{k} \int_{0}^{T} \phi_{l}(y) d B_{l}^{H}(y)$. We will first prove by induction that

$$
\begin{aligned}
& E\left|\sum_{l=1}^{k} \int_{0}^{T} \phi_{l}(y) d B_{l}^{H}(y)\right|^{2} \\
& \quad \leq H(2 H-1) \sum_{l=1}^{k} \int_{0}^{T} \int_{0}^{T}\left\|\phi_{l}(y)\right\|_{L_{Q}^{0}}\left\|\phi_{l}(z)\right\|_{L_{Q}^{0}}|y-z|^{2 H-2} d y d z
\end{aligned}
$$

holds for all $k \in \mathbb{N}$.

Observe that if $k=1$ we have

$$
\begin{aligned}
E\left|M^{1}\right|_{X}^{2} & =E\left|\int_{0}^{T} \phi_{1}(y) d B_{1}^{H}(y)\right|_{X}^{2} \\
& =E \sum_{n=1}^{\infty}\left|\int_{0}^{T} \phi_{1}(y) Q^{\frac{1}{2}} e_{n} d \beta_{1, n}^{H}(y)\right|_{X}^{2} \\
& =E \sum_{n=1}^{\infty}\left\langle\int_{0}^{T} \phi_{1}(y) Q^{\frac{1}{2}} e_{n} d \beta_{1, n}^{H}(y), \int_{0}^{T} \phi_{1}(y) Q^{\frac{1}{2}} e_{n} d \beta_{1, n}^{H}(y)\right\rangle \\
& =E \sum_{n=1}^{\infty}\left\langle\int_{0}^{T}\left(K_{H}^{*}\left(\phi Q^{\frac{1}{2}} e_{n}\right)\right)(s) d \beta_{1, n}(s), \int_{0}^{T}\left(K_{H}^{*}\left(\phi Q^{\frac{1}{2}} e_{n}\right)\right)(s) d \beta_{1, n}(s)\right\rangle .
\end{aligned}
$$

Then

$$
\begin{aligned}
& E\left|\int_{0}^{T} \phi_{1}(y) d B_{1}^{H}(y)\right|_{X}^{2} \\
& \quad \leq H(2 H-1) \int_{0}^{T} \int_{0}^{T}\left\|\phi_{1}(y)\right\|_{L_{Q}^{0}}\left\|\phi_{1}(z)\right\|_{L_{Q}^{0}}|y-z|^{2 H-2} d y d z .
\end{aligned}
$$

Therefore the result holds for $k=1$. 
Suppose now that (2.11) holds for a fixed integer number $k>1$, and let us prove it for $k+1$. Indeed, since $\left(\phi_{l}\right)_{l=1}^{k+1}$ is a set of elementary stochastic process, and on account of the induction hypothesis we have

$$
\begin{aligned}
E\left|M^{k+1}\right|_{X}^{2} & =E\left|\sum_{l=1}^{k+1} \int_{0}^{T} \phi_{l}(y) d B_{l}^{H}(y)\right|_{X}^{2} \\
& =E\left|\sum_{l=1}^{k} \int_{0}^{T} \phi_{l}(y) d B_{l}^{H}(y)\right|_{X}^{2}+E\left|\int_{0}^{T} \phi_{k+1}(y) d B_{k+1}^{H}(y)\right|_{X}^{2} \\
& +2 E\left\langle\sum_{l=1}^{k} \int_{0}^{T} \phi_{l}(y) d B_{l}^{H}(y), \int_{0}^{T} \phi_{k+1}(y) d B_{k+1}^{H}(y)\right\rangle \\
& \leq \sum_{l=1}^{k} H(2 H-1) \int_{0}^{T} \int_{0}^{T}\left\|\phi_{l}(y)\right\|_{L_{Q}^{0}}\left\|\phi_{l}(z)\right\|_{L_{Q}^{0}}|y-z|^{2 H-2} d y d z \\
& +H(2 H-1) \int_{0}^{T} \int_{0}^{T}\left\|\phi_{k+1}(y)\right\|_{L_{Q}^{0}}\left\|\phi_{k+1}(z)\right\|_{L_{Q}^{0}}|y-z|^{2 H-2} d y d z \\
& +2 E\left\langle\sum_{l=1}^{k} \int_{0}^{T} \phi_{l}(y) d B_{l}^{H}(y), \int_{0}^{T} \phi_{k+1}(y) d B_{k+1}^{H}(y)\right\rangle \\
& =\sum_{l=1}^{k} H(2 H-1) \int_{0}^{T} \int_{0}^{T}\left\|\phi_{l}(y)\right\|_{L_{Q}^{0}}\left\|\phi_{l}(z)\right\|_{L_{Q}^{0}}|y-z|^{2 H-2} d y d z \\
& +H(2 H-1) \int_{0}^{T} \int_{0}^{T}\left\|\phi_{k+1}(y)\right\|_{L_{Q}^{0}}\left\|\phi_{k+1}(z)\right\|_{L_{Q}^{0}}|y-z|^{2 H-2} d y d z \\
& +2 E\left\langle\sum_{l=1}^{k} \sum_{k=1}^{n} \phi_{k}\left(B_{l}^{H}\left(\bar{t}_{k}\right)^{H}-B_{l}^{H}\left(\bar{t}_{k-1}\right)\right), \sum_{i=1}^{n} \phi_{i}\left(B_{k+1}^{H}\left(\bar{t}_{i}\right)-B_{k+1}^{H}\left(\bar{t}_{i-1}\right)\right)\right\rangle .
\end{aligned}
$$

Thanks to $(2.11)$ and the fact that $\left(B_{l}^{H}\right)_{l=1}^{k+1}$ is a set of independent standard fractional Brownian motions, it follows

$E\left|\sum_{l=1}^{k+1} \int_{0}^{T} \phi_{l}(y) d B_{l}^{H}(y)\right|_{X}^{2} \leq \sum_{l=1}^{k+1} H(2 H-1) \int_{0}^{T} \int_{0}^{T}\left\|\phi_{l}(y)\right\|_{L_{Q}^{0}}\left\|\phi_{l}(z)\right\|_{L_{Q}^{0}}|y-z|^{2 H-2} d y d z$,

and hence the formula is true for $k+1$ as we wished. From Corollary 2.1, we know that $M^{k}$ is convergent in $L^{2}\left(\Omega, \mathcal{F}_{t}, \mathbb{P} ; X\right)$, and thanks to the continuity of the norm,

$$
\begin{aligned}
E\left|\lim _{k \rightarrow \infty} M^{k}\right|_{X}^{2} & =\lim _{k \rightarrow \infty} E\left|\sum_{l=1}^{k} \int_{0}^{T} \phi_{l}(y) d B_{l}^{H}(y)\right|_{X}^{2} \\
& \leq \sum_{l=1}^{\infty} H(2 H-1) \int_{0}^{T} \int_{0}^{T}\left\|\phi_{l}(y)\right\|_{L_{Q}^{0}}\left\|\phi_{l}(z)\right\|_{L_{Q}^{0}}|y-z|^{2 H-2} d y d z
\end{aligned}
$$


and, consequently,

$E\left|\sum_{l=1}^{\infty} \int_{0}^{T} \phi_{l}(y) d B_{l}^{H}(y)\right|_{X}^{2} \leq \sum_{l=1}^{\infty} H(2 H-1) \int_{0}^{T} \int_{0}^{T}\left\|\phi_{l}(y)\right\|_{L_{Q}^{0}}\left\|\phi_{l}(z)\right\|_{L_{Q}^{0}}|y-z|^{2 H-2} d y d z$.

\section{$3 \quad$ Fixed point results}

Let us recall in this section some useful definitions and results concerning fixed point theorems. It is worth mentioning that the classical Banach contraction principle was extended to contractive maps on spaces endowed with vector-valued metric space by Perov [29] in 1964, Perov and Kibenko [31] and Precup [30].

For $x, y \in \mathbb{R}^{n}, x=\left(x_{1}, \ldots, x_{n}\right), y=\left(y_{1}, \ldots, y_{n}\right)$, by $x \leq y$ we mean $x_{i} \leq y_{i}$ for all $i=1, \ldots, n$. Also $|x|=\left(\left|x_{1}\right|, \ldots,\left|x_{n}\right|\right)$ and $\max (x, y)=\max \left(\max \left(x_{1}, y_{1}\right), \ldots, \max \left(x_{n}, y_{n}\right)\right)$. If $c \in \mathbb{R}$, then $x \leq c$ means $x_{i} \leq c$ for each $i=1, \ldots, n$.

Definition 3.1. Let $X$ be a nonempty set. A vector-valued metric on $X$ is a map $d: X \times X \rightarrow \mathbb{R}^{n}$ with the following properties:

(i) $d(u, v) \geq 0$ for all $u, v \in X$; if $d(u, v)=0$ then $u=v$;

(ii) $d(u, v)=d(v, u)$ for all $u, v \in X$;

(iii) $d(u, v) \leq d(u, w)+d(w, v)$ for all $u, v, w \in X$.

The pair $(X, d)$ a said to be a generalized metric space.

For $r=\left(r_{1}, \ldots, r_{n}\right) \in \mathbb{R}_{+}^{n}$, we will denote by

$$
B\left(x_{0}, r\right)=\left\{x \in X: d\left(x_{0}, x\right)<r\right\}
$$

the open ball centered in $x_{0}$ with radius $r$ and

$$
\overline{B\left(x_{0}, r\right)}=\left\{x \in X: d\left(x_{0}, x\right) \leq r\right\}
$$

the closed ball centered in $x_{0}$ with radius $r$. We mention that for generalized metric space, the notation of open subset, closed set, convergence, Cauchy sequence and completeness are similar to those in usual metric spaces.

Definition 3.2. A generalized metric space $(X, d)$, where $d(x, y):=\left(\begin{array}{c}d_{1}(x, y) \\ \cdots \\ d_{n}(x, y)\end{array}\right)$, is complete if $\left(X, d_{i}\right)$ is a complete metric space for every $i=1, \ldots, n$. 
Definition 3.3. A square matrix of real numbers is said to be convergent to zero if its spectral radius $\rho(M)$ is strictly less than 1 . In other words, this means that all the eigenvalues of $M$ are in the open unit disc. (i.e. $|\lambda|<1$, for every $\lambda \in \mathbb{C}$ with $\operatorname{det}(M-\lambda I)=0$, where $I$ denote the unit matrix of $\left.\mathcal{M}_{n \times n}(\mathbb{R})\right)$.

Definition 3.4. A non-singular matrix $A=\left(a_{i j}\right)_{1 \leq i, j \leq n} \in \mathcal{M}_{n \times n}(\mathbb{R})$ is said to have the absolute value property if

$$
A^{-1}|A| \leq I
$$

where

$$
|A|=\left(\left|a_{i j}\right|\right)_{1 \leq i, j \leq n} \in \mathcal{M}_{n \times n}\left(\mathbb{R}_{+}\right) .
$$

Lemma 3.1. [33] Let $M$ be a square matrix of nonnegative numbers. The following assertions are equivalent:

(i) $M$ is convergent towards zero;

(ii) the matrix $I-M$ is non-singular and

$$
(I-M)^{-1}=I+M+M^{2}+\ldots+M^{k}+\ldots ;
$$

(iii) $|\lambda|<1$ for every $\lambda \in \mathbb{C}$ with $\operatorname{det}(M-\lambda I)=0$

(iv) $(I-M)$ is non-singular and $(I-M)^{-1}$ has nonnegative elements;

Some examples of matrices convergent to zero are the following:

1) $A=\left(\begin{array}{cc}a & 0 \\ 0 & b\end{array}\right)$, where $a, b \in \mathbb{R}_{+}$and $\max (a, b)<1$

2) $A=\left(\begin{array}{ll}a & -c \\ 0 & b\end{array}\right)$, where $a, b, c \in \mathbb{R}_{+}$and $a+b<1, c<1$

3) $A=\left(\begin{array}{cc}a & -a \\ b & -b\end{array}\right)$, where $a, b, c \in \mathbb{R}_{+}$and $|a-b|<1, a>1, b>0$.

We recall now a fixed point theorem in a complete generalized metric space due to Perov.

Theorem 3.1. [29] Let $(X, d)$ be a complete generalized metric space with $d: X \times X \rightarrow$ $\mathbb{R}^{n}$ and let $N: X \rightarrow X$ be a mapping such that

$$
d(N(x), N(y)) \leq M d(x, y)
$$

for all $x, y \in X$ and some square matrix $M$ of nonnegative numbers. If the matrix $M$ is convergent to zero, that is $M^{k} \rightarrow 0$ as $k \rightarrow \infty$, then $N$ has a unique fixed point $x_{*} \in X$ such that

$$
d\left(N^{k}\left(x_{0}\right), x_{*}\right) \leq M^{k}(I-M)^{-1} d\left(N\left(x_{0}\right), x_{0}\right)
$$

for every $x_{0} \in X$ and $k \geq 1$. 
Next we state the version of nonlinear alternative Leary-Schauder type theorem in generalized Banach space [4].

Theorem 3.2. Let $C \subset E$ be a closed convex subset and $U \subset C$ a bounded open neighborhood of zero (with respect to topology of $C$ ). If $N: \bar{U} \rightarrow E$ is compact continuous then

i) either $N$ has a fixed point in $\bar{U}$, or

ii) there exists $x \in \partial U$ such that $x=\lambda N(x)$ for some $\lambda \in(0,1)$.

\section{Existence and uniqueness of mild solution}

Let $J_{k}=\left(t_{k}, t_{k+1}\right], k=1,2, \ldots, m$. In order to define a solution for Problem (1.1), consider the following space of pice-wise continuous functions

$$
\begin{aligned}
P C= & \left\{x: \Omega \times[0, T] \longrightarrow X, x \in C\left(J_{k}, L^{2}(\Omega, X)\right), k=1, \ldots, m\right. \text { such that } \\
& x\left(t_{k}^{+}, .\right) \text {and } x\left(t_{k}^{-}, .\right) \text {exist with } x\left(t_{k}^{-}, .\right)=x\left(t_{k}, .\right) \text { and } \\
& \left.\sup _{t \in[0, T]} E\|x(t, .)\|^{2}<\infty \text { almost surely }\right\}
\end{aligned}
$$

endowed with the norm

$$
\|x\|_{P C}=\sup _{s \in[0, T]}\left(E\|x(s, .)\|^{2}\right)^{\frac{1}{2}} .
$$

It is not difficult to check that $P C$ is a Banach space with norm $\|\cdot\|_{P C}$.

First, we will list the following hypotheses which will be imposed in our main theorem. In this section, we assume that there exists $M>0$ such that

$$
\|S(t)\| \leq M, \quad \text { for every } t \in[0, T] .
$$

$\left(H_{1}\right)$ There exist functions $a_{i}, b_{i} \in L^{1}\left([0, T], \mathbb{R}^{+}\right)$such that

$$
\left|f^{i}(t, x, y)-f^{i}(t, \bar{x}, \bar{y})\right|_{X}^{2} \leq a_{i}(t)|x-\bar{x}|_{X}^{2}+b_{i}(t)|y-\bar{y}|_{X}^{2}, \quad i=1,2
$$

for all $x, y, \bar{x}, \bar{y} \in X$.

$\left(H_{2}\right)$ There exist functions $\alpha_{i}, \beta_{i} \in L^{1}\left([0, T], \mathbb{R}^{+}\right)$such that

$$
\left\|\sigma^{i}(t, x, y)-\sigma^{i}(t, \bar{x}, \bar{y})\right\|^{2} \leq \alpha_{i}(t)|x-\bar{x}|_{X}^{2}+\beta_{i}(t)|y-\bar{y}|_{X}^{2}
$$

and

The function $\sigma: J \times X \times X \longrightarrow L_{Q}^{0}(Y, X)$ satisfies

$$
\sum_{l=1}^{\infty} \int_{0}^{T}\left\|\sigma_{l}^{i}(s, x(s), y(s))\right\|_{L_{Q}^{0}}^{2} d s<\infty .
$$

for all $x, y, \bar{x}, \bar{y} \in X$ and a.e $t \in J$. 
Now, we first define the concept of mild solution to our problem.

Definition 4.1. An $X$-valued stochastic process $u=(x, y) \in P C \times P C$ is said to be a solution of $(1.1)$ with respect to the probability space $(\Omega, \mathcal{F}, \mathbb{P})$, if:

1) $u(t)$ is $\mathcal{F}_{t}$-adapted for all $t \in J_{k}=\left(t_{k}, t_{k+1}\right] \quad k=1,2, \ldots, m$

2) $u(t)$ is right continuous and has limit on the left almost surely;

3) $u(0)=\left(x_{0}, y_{0}\right)$

4) $u(t)$ satisfies for all $t \in[0, T]$ and almost surely that

$$
\left\{\begin{aligned}
x(t)= & S(t) x_{0}+\int_{0}^{t} S(t-s) f^{1}(s, x(s), y(s)) d s \\
+ & \sum_{l=1}^{\infty} \int_{0}^{t} S(t-s) \sigma_{l}^{1}(t, x(s), y(s)) d B_{l}^{H}(s) \\
& +\sum_{0<t_{k}<t} S\left(t-t_{k}\right) I_{k}\left(x\left(t_{k}\right)\right), \quad \mathbb{P}-a . s, \quad t \in J \\
y(t)= & S(t) y_{0}+\int_{0}^{t} S(t-s) f^{2}(s, x(s), y(s)) d s \\
+ & \sum_{l=1}^{\infty} \int_{0}^{t} S(t-s) \sigma_{l}^{2}(t, x(s), y(s)) d B_{l}^{H}(s) \\
& +\sum_{0<t_{k}<t} S\left(t-t_{k}\right) I_{k}\left(y\left(t_{k}\right)\right), \quad \mathbb{P}-a . s, \quad t \in J .
\end{aligned}\right.
$$

Notice that, thanks to (4.1) and the fact that $H \in(1 / 2,1),(2.6)$ holds, which implies that the stochastic integrals in $(4.2)$ are well-defined since $S(\cdot)$ is a strongly continuous semigroup, for every $t \in[0, T]$, and that this concept of solution can be considered as more general than the classical concept of solution to equation (1.1). A continuous solution of (4.2) is called a mild solution of (1.1).

Definition 4.2. The map $f: J \times X \rightarrow X$ is said to be $L^{2}$-Caratheodory if

i) $t \mapsto f(t, u)$ is measurable for each $u \in X$;

ii) $u \mapsto f(t, u)$ is continuous for almost all $t \in J$;

iii) for each $q>0$, there exists $\alpha_{q} \in L^{1}\left(J, \mathbb{R}^{+}\right)$such that

$E|f(t, u)|_{X}^{2} \leq \alpha_{q}$, for all $u \in X$ such that $E|u|_{X}^{2} \leq q$ and for a.e. $t \in J$.

Let us now prove the existence and uniqueness of mild solution for (1.1) by using Perov's fixed point theorem. 
Theorem 4.1. Assume that $\left(H_{1}\right)$ and $\left(H_{2}\right)$ hold. Then, problem (1.1) possesses a unique mild solution on $[0, T]$.

Proof. The proof will be split into several steps.

Step 1. Consider the problem

$$
\left\{\begin{aligned}
d x(t) & =\left(A x(t)+f^{1}(t, x(t), y(t)) d t\right. \\
& \left.+\sum_{l=1}^{\infty} \sigma_{l}^{1}(t, x(t)), y(t)\right) d B_{l}^{H}(t), t \in\left[0, t_{1}\right] \\
d y(t) & =\left(A y(t)+f^{2}(t, x(t), y(t))\right) d t \\
& +\sum_{l=1}^{\infty} \sigma_{l}^{2}(t, x(t), y(t)) d B_{l}^{H}(t), t \in\left[0, t_{1}\right] \\
x(0) & =x_{0} \\
y(0) & =y_{0}
\end{aligned}\right.
$$

Let

$$
D_{t_{0}}=\left\{x: \Omega \times\left[0, t_{1}\right] \longrightarrow X, x \in C\left(J_{1}, L^{2}(\Omega, X)\right): \sup _{t \in\left[0, t_{1}\right]} E|x(t, \cdot)|_{X}^{2}<\infty\right\},
$$

Consider the operator

$$
P^{0}: D_{t_{0}} \times D_{t_{0}} \rightarrow D_{t_{0}} \times D_{t_{0}}
$$

defined by

$$
P^{0}(x, y)=\left(P_{1}^{0}(x, y), P_{2}^{0}(x, y)\right),(x, y) \in D_{t_{0}} \times D_{t_{0}}
$$

where

$$
\left\{\begin{aligned}
P_{1}^{0}(x, y)= & S(t) x_{0}+\int_{0}^{t} S(t-s) f^{1}(s, x(s), y(s)) d s \\
& +\sum_{l=1}^{\infty} \int_{0}^{t} S(t-s) \sigma_{l}^{1}(t, x(s), y(s)) d B_{l}^{H}(s), \quad t \in\left[0, t_{1}\right] . \\
P_{2}^{0}(x, y)= & S(t) y_{0}+\int_{0}^{t} S(t-s) f^{2}(s, x(s), y(s)) d s \\
& +\sum_{l=1}^{\infty} \int_{0}^{t} S(t-s) \sigma_{l}^{2}(t, x(s), y(s)) d B_{l}^{H}(s), \quad t \in\left[0, t_{1}\right] .
\end{aligned}\right.
$$

The operators in (4.4) are well-defined. In other words, given $(x, y) \in D_{t_{0}} \times D_{t_{0}}$, we see that $P^{0}(x, y) \in D_{t_{0}} \times D_{t_{0}}$ as well. We will use Theorem 3.1 to prove that $P^{0}$ 
possesses a fixed point. Let $(x, y),(\bar{x}, \bar{y}) \in D_{t_{0}} \times D_{t_{0}}$, then for each $t \in\left[0, t_{1}\right]$ we have

$$
\begin{aligned}
& \left|P_{1}^{0}(x(t), y(t))-P_{1}^{0}(\bar{x}(t), \bar{y}(t))\right|_{X}^{2} \\
& =\mid \int_{0}^{t} S(t-s) f^{1}(s, x(s), y(s)) d s+\sum_{l=1}^{\infty} \int_{0}^{t} S(t-s) \sigma_{l}^{1}(t, x(s), y(s)) d B_{l}^{H}(s) \\
& \quad-\int_{0}^{t} S(t-s) f^{1}(s, \bar{x}(s), \bar{y}(s)) d s-\left.\sum_{l=1}^{\infty} \int_{0}^{t} S(t-s) \sigma_{l}^{1}(s, \bar{x}(s), \bar{y}(s)) d B_{l}^{H}(s)\right|_{X} ^{2} \\
& \leq 2\left|\int_{0}^{t} S(t-s)\left(f^{1}(s, x(s), y(s))-f^{1}(s, \bar{x}(s), \bar{y}(s))\right) d s\right|_{X}^{2} \\
& \quad+2\left|\sum_{l=1}^{\infty} \int_{0}^{t} S(t-s)\left(\sigma_{l}^{1}(s, x(s), y(s))-\sigma_{l}^{1}(s, \bar{x}(s), \bar{y}(s))\right) d B_{l}^{H}(s)\right|_{X}^{2} .
\end{aligned}
$$

Lemma 2.3 and assumptions $\left(H_{1}\right),\left(H_{2}\right)$ imply

$$
\begin{aligned}
E \mid P_{1}^{0}( & x(t), y(t))-\left.P_{1}^{0}(\bar{x}(t), \bar{y}(t))\right|_{X} ^{2} \\
\leq & 2 M^{2} t_{1} \int_{0}^{t} a_{1}(s) E|x(s)-\bar{x}(s)|_{X}^{2}+b_{1}(s) E|y(s)-\bar{y}(s)|_{X}^{2} d s \\
& 2 c_{H} H(2 H-1) t_{1}^{2 H-1} \int_{0}^{t} M^{2} d s \int_{0}^{t} \alpha_{1}(s) E|x(s)-\bar{x}(s)|_{X}^{2} d s \\
& \quad+2 c_{H} H(2 H-1) t_{1}^{2 H-1} \int_{0}^{t} M^{2} d s \int_{0}^{t} \beta_{1}(s) E|y(s)-\bar{y}(s)|_{X}^{2} d s \\
\leq & \int_{0}^{t} \alpha(s) e^{\tau \widehat{\alpha}(s)} e^{-\tau \widehat{\alpha}(s)} \sup _{s \in\left[0, t_{1}\right]} E|x(s)-\bar{x}(s)|_{X}^{2} d s \\
& +\int_{0}^{t} \alpha(s) e^{\tau \widehat{\alpha}(s)} e^{-\tau \widehat{\alpha}(s)} \sup _{s \in\left[0, t_{1}\right]} E|y(s)-\bar{y}(s)|_{X}^{2} d s \\
\leq & \int_{0}^{t} \alpha(s) e^{\tau \widehat{\alpha}(s)} d s\|x-\bar{x}\|_{*}^{2}+\int_{0}^{t} \alpha(s) e^{\tau \widehat{\alpha}(s)} d s\|y-\bar{y}\|_{*}^{2} \\
\leq & \frac{1}{\tau} \int_{0}^{t}\left(e^{\tau \widehat{\alpha}(s)}\right)^{\prime} d s\|x-\bar{x}\|_{*}^{2}+\frac{1}{\tau} \int_{0}^{t}\left(e^{\tau \widehat{\alpha}(s)}\right)^{\prime} d s\|y-\bar{y}\|_{*}^{2} \\
\leq & \frac{1}{\tau} e^{\tau \widehat{\alpha}(t)}\|x-\bar{x}\|_{*}^{2}+\frac{1}{\tau} e^{\tau \widehat{\alpha}(t)}\|y-\bar{y}\|_{*}^{2} .
\end{aligned}
$$

Therefore

$$
e^{-\tau \widehat{\alpha}(t)} E\left|L_{1}(x(t), y(t))-L_{1}(\bar{x}(t), \bar{y}(t))\right|_{X}^{2} \leq \frac{1}{\tau}\|x-\bar{x}\|_{*}^{2}+\frac{1}{\tau}\|y-\bar{y}\|_{*}^{2},
$$

where $\|\cdot\|_{*}$ is the Bielecki-type norm on $D_{t_{0}}$ defined by

$$
\|x\|_{*}^{2}=\sup _{t \in\left[0, t_{1}\right]} E|x(t, .)|_{X}^{2} e^{-\tau \widehat{\alpha}(t)}
$$

where

$$
\widehat{\alpha}(t)=\int_{0}^{t} \alpha(s) d s, \quad t \in\left[0, t_{1}\right],
$$


and

$\alpha(s)=\max \left\{2 M^{2} t_{1} a_{1}(s)+2 c_{H} H(2 H-1) t_{1}^{2 H} M^{2} \alpha_{1}(s), 2 M^{2} t_{1} b_{1}(s)+2 c_{H} H(2 H-1) t_{1}^{2 H} M^{2} \beta_{1}(s)\right\}$.

Hence

$$
\left\|P_{1}^{0}(x, y)-P_{1}^{0}(\bar{x}, \bar{y})\right\|_{*}^{2} \leq \frac{1}{\tau}\|x-\bar{x}\|_{*}^{2}+\frac{1}{\tau}\|y-\bar{y}\|_{*}^{2} .
$$

Using the fact that for all $a, b \geq 0$ we have $\sqrt{a+b} \leq \sqrt{a}+\sqrt{b}$, we conclude that

$$
\left\|P_{1}^{0}(x, y)-P_{1}^{0}(\bar{x}, \bar{y})\right\|_{*} \leq \frac{1}{\sqrt{\tau}}\|x-\bar{x}\|_{*}+\frac{1}{\sqrt{\tau}}\|y-\bar{y}\|_{*} .
$$

Similar computations for $N_{1}$ yield

$$
\left\|P_{2}^{0}(x, y)-P_{2}^{0}(\bar{x}, \bar{y})\right\|_{*} \leq \frac{1}{\sqrt{\tau}}\|x-\bar{x}\|_{*}+\frac{1}{\sqrt{\tau}}\|y-\bar{y}\|_{*} .
$$

Thus

$$
\begin{aligned}
\left\|P^{0}(x, y)-P^{0}(\bar{x}, \bar{y})\right\|_{*} & =\left(\begin{array}{l}
\| P_{1}^{0}\left((x, y)-P_{1}^{0}(\bar{x}, \bar{y}) \|_{*}\right. \\
\left\|P_{2}^{0}(x, y)-P_{2}^{0}(\bar{x}, \bar{y})\right\|_{*}
\end{array}\right) \\
& \leq \frac{1}{\sqrt{\tau}}\left(\begin{array}{ll}
1 & 1 \\
1 & 1
\end{array}\right)\left(\begin{array}{l}
\|x-\bar{x}\|_{*} \\
\|y-\bar{y}\|_{*}
\end{array}\right) .
\end{aligned}
$$

Hence

$$
\left\|P^{0}(x, y)-P^{0}(\bar{x}, \bar{y})\right\|_{*} \leq \frac{1}{\sqrt{\tau}} M_{\alpha, \beta}\left(\begin{array}{l}
\|x-\bar{x}\|_{*} \\
\|y-\bar{y}\|_{*}
\end{array}\right),
$$

for all $(x, y),(\bar{x}, \bar{y}) \in D_{t_{0}} \times D_{t_{0}}$, where

$$
M_{\alpha, \beta}=\left(\begin{array}{ll}
1 & 1 \\
1 & 1
\end{array}\right)
$$

If we choose a suitable $\sqrt{\tau}>2$ such that the matrix

$$
\frac{\left\|M_{\alpha, \beta}\right\|}{\sqrt{\tau}}<1
$$

then $\frac{M_{\alpha, \beta}}{\tau}$ is nonnegative, $I-\frac{\left\|M_{\alpha, \beta}\right\|}{\tau}$ is non singular and

$$
\left(I-\frac{M_{\alpha, \beta}}{\sqrt{\tau}}\right)^{-1}=I+\frac{M_{\alpha, \beta}}{\sqrt{\tau}}+\frac{M_{\alpha, \beta}^{2}}{\tau}+\ldots
$$

From Lemma 3.1, we obtain that $\frac{M_{\alpha, \beta}}{\sqrt{\tau}}$ converges to zero. As a consequence of Perov's fixed point theorem, $P^{0}$ has a unique fixed $(x, y) \in D_{t_{0}} \times D_{t_{0}}$ which is the unique solution of problem (4.3). Let us denote this solution by $\left(x_{1}, y_{1}\right)$. 
Step 2. Now consider the problem

$$
\left\{\begin{aligned}
d x(t) & =\left(A x(t)+f^{1}(t, x(t), y(t)) d t\right. \\
& \left.+\sum_{l=1}^{\infty} \sigma_{l}^{1}(t, x(t)), y(t)\right) d B_{l}^{H}(t), t \in\left(t_{1}, t_{2}\right] \\
d y(t) & =\left(A y(t)+f^{2}(t, x(t), y(t))\right) d t \\
& +\sum_{l=1}^{\infty} \sigma_{l}^{2}(t, x(t), y(t)) d B_{l}^{H}(t), t \in\left(t_{1}, t_{2}\right] \\
x\left(t_{1}^{+}\right) & =x_{1}\left(t_{1}^{-}\right)+I_{1}\left(x_{1}\left(t_{1}\right)\right), \\
y\left(t_{1}^{+}\right) & =y_{1}\left(t_{1}^{-}\right)+\bar{I}_{1}\left(y_{1}\left(t_{1}\right)\right) .
\end{aligned}\right.
$$

Let

$$
D_{t_{1}}=\left\{x \in C\left(\left(t_{1}, t_{2}\right], L^{2}(\Omega, X)\right) \text { such that } x\left(t_{1}^{+}, .\right) \text {and } x\left(t_{1}^{-}, .\right) \text {exist with } x\left(t_{1}^{-}, .\right)=x\left(t_{1}, .\right)\right. \text { and }
$$

Set

$$
C_{1}=D_{t_{0}} \cap D_{t_{1}}
$$

Consider the operator $P: C_{1} \times C_{1} \rightarrow C_{1} \times C_{1}$ defined by

$$
P^{1}(x, y)=\left(P_{1}^{1}(x, y), P_{2}^{1}(x, y)\right),(x, y) \in C_{1} \times C_{1}
$$

where

$$
\left\{\begin{aligned}
P_{1}^{1}(x, y)= & S\left(t-t_{1}\right)\left[x_{1}\left(t_{1}\right)+I_{1}\left(x_{1}\left(t_{1}^{-}\right)\right)\right]+\int_{t_{1}}^{t} S(t-s) f^{1}(s, x(s), y(s)) d s \\
& +\sum_{l=1}^{\infty} \int_{t_{1}}^{t} S(t-s) \sigma_{l}^{1}(t, x(s), y(s)) d B_{l}^{H}(s), \quad t \in\left[t_{1}, t_{2}\right] . \\
P_{2}^{1}(x, y)= & S\left(t-t_{1}\right)\left[y_{1}\left(t_{1}\right)+I_{1}\left(y_{1}\left(t_{1}^{-}\right)\right)\right]+\int_{t_{1}}^{t} S(t-s) f^{2}(s, x(s), y(s)) d s \\
& +\sum_{l=1}^{\infty} \int_{t_{1}}^{t} S(t-s) \sigma_{l}^{2}(t, x(s), y(s)) d B_{l}^{H}(s), \quad t \in\left[t_{1}, t_{2}\right] .
\end{aligned}\right.
$$

As in Step 1, we can show that $P^{1}$ is well defined and the integral equation possesses a unique fixed point $(x, y)$ which is a solution to problem (4.5). Denote this solution by $\left(x_{2}, y_{2}\right)$.

Step 3 We continue this process taking into account that $\left(x_{m}, y_{m}\right)=\left(\left.x\right|_{\left[t_{m}, T\right]},\left.y\right|_{\left[t_{m}, T\right]}\right)$ 
is a solution of the problem

$$
\left\{\begin{aligned}
d x(t) & =\left(A x(t)+f^{1}(t, x(t), y(t)) d t\right. \\
& \left.+\sum_{l=1}^{\infty} \sigma_{l}^{1}(t, x(t)), y(t)\right) d B_{l}^{H}(t), t \in\left(t_{m}, T\right], \\
d y(t) & =\left(A y(t)+f^{2}(t, x(t), y(t))\right) d t \\
& +\sum_{l=1}^{\infty} \sigma_{l}^{2}(t, x(t), y(t)) d B_{l}^{H}(t), t \in\left(t_{m}, T\right], \\
x\left(t_{m}^{+}\right) & =x_{m}\left(t_{m}^{-}\right)+I_{m}\left(x_{m}\left(t_{m}\right)\right) \\
y\left(t_{m}^{+}\right) & =y_{m}\left(t_{m}^{-}\right)+\bar{I}_{m}\left(y_{m}\left(t_{1}\right)\right) .
\end{aligned}\right.
$$

Let

$$
D_{t_{m}}=\left\{x \in C\left(\left(t_{m}, T\right], L^{2}(\Omega, X)\right) \text { such that } x\left(t_{1}^{+}, .\right) \text {and } x\left(t_{1}^{-}, .\right) \text {exist with } x\left(t_{m}^{-}, .\right)=x\left(t_{m}, .\right)\right. \text { and }
$$

Set

$$
C_{m}=\cap_{k=0}^{m} D_{t_{k}}
$$

Then, there exists a fixed point $\left(x_{m}(t), y_{m}(t)\right)$ of $P^{m}=\left(P_{1}^{m}, P_{2}^{m}\right): C_{m} \times C_{m} \rightarrow C_{m} \times C_{m}$. The unique solution $(x, y)$ of problem $(1.1)$ is then defined by

$$
\left\{\begin{aligned}
P_{1}^{m}(x, y)= & S\left(t-t_{m}\right)\left[x_{m}\left(t_{m}\right)+I_{m}\left(x_{1}\left(t_{m}^{-}\right)\right)\right]+\int_{t_{m}}^{t} S(t-s) f^{1}(s, x(s), y(s)) d s \\
& +\sum_{l=1}^{\infty} \int_{t_{m}}^{t} S(t-s) \sigma_{l}^{1}(t, x(s), y(s)) d B_{l}^{H}(s), \quad t \in\left[t_{m}, T\right] . \\
P_{2}^{m}(x, y)= & S\left(t-t_{m}\right)\left[y_{m}\left(t_{m}\right)+I_{m}\left(y_{1}\left(t_{m}^{-}\right)\right)\right]+\int_{t_{m}}^{t} S(t-s) f^{2}(s, x(s), y(s)) d s \\
& +\sum_{l=1}^{\infty} \int_{t_{m}}^{t} S(t-s) \sigma_{l}^{2}(t, x(s), y(s)) d B_{l}^{H}(s), \quad t \in\left[t_{m}, T\right] . \\
& (x(t), y(t))= \begin{cases}\left(x_{1}(t), y_{1}(t)\right), & \text { if, } t \in\left[0, t_{1}\right], \\
\left(x_{2}(t), y_{2}(t)\right), & \text { if, } t \in\left(t_{1}, t_{2}\right], \\
\cdots & \text { if, } t \in\left(t_{m}, T\right] \\
\left(x_{m+1}(t), y_{m+1}(t)\right),\end{cases}
\end{aligned}\right.
$$

and the proof is finished.

The second result in this section dealing with the existence of solutions to our problem will be obtained by applying the Leary-Schauder fixed point theorem. To this end we first need to introduce the following hypotheses: 
$\left(H_{3}\right)$ There exist functions $\bar{a}_{i}, \bar{b}_{i}, c_{i} \in L^{1}\left([0, T], \mathbb{R}^{+}\right)$such that each

$$
\left|f^{i}(t, x, y)\right|_{X}^{2} \leq \bar{a}_{i}(t)|x|_{X}^{2}+\bar{b}_{i}(t)|y|_{X}^{2}+c_{i}(t), i=1,2 .
$$

for all $x, y \in X$, and a.e. $t \in J$.

$\left(H_{4}\right)$ There exist functions $\bar{\alpha}_{i} \in L^{1}\left([0, T], \mathbb{R}^{+}\right)$and $\bar{\beta}_{i}, \bar{c}_{i} \in L^{1}\left([0, T], \mathbb{R}^{+}\right)$such that

$$
\left\|\sigma^{i}(t, x, y)\right\|^{2} \leq \bar{\alpha}_{i}(t)|x|_{X}^{2}+\bar{\beta}_{i}(t)|y|_{X}^{2}+\bar{c}_{i}(t), \quad i=1,2
$$

for all $x, y \in X$, and a.e. $t \in J$.

$\left(H_{5}\right)$ The semigroup $\{S(t)\}_{t>0}$ is compact in $X$

$\left(H_{6}\right)$ there exist constants $d_{k}, \lambda_{k} \geq 0$ and $\bar{d}_{k}, \bar{\lambda}_{k} \geq 0, k=1, \ldots, m$ such that

$$
\begin{aligned}
\left|I_{k}(x)\right|_{X}^{2} & \leq d_{k}|x|_{X}^{2}+\lambda_{k} \\
\left|\bar{I}_{k}(y)\right|^{2} & \leq \bar{d}_{k}|y|_{X}^{2}+\bar{\lambda}_{k}
\end{aligned}
$$

for all $x, y \in X$.

$\left(H_{7}\right) f^{i}, g^{i}$ are $L^{2}$-Carathéodory maps.

We now prove our second goal of this section.

Theorem 4.2. Assume conditions $\left(H_{3}\right)-\left(H_{7}\right)$ hold. Then, problem (1.1) has at least one solution.

Proof. We transform problem (4.3) into a fixed point problem (4.3). Consider the operator $N: P C \times P C \rightarrow P C \times P C$ defined by

$$
N(x, y)=\left(N_{1}(x, y), N_{2}(x, y)\right),(x, y) \in P C \times P C
$$

where

$$
\left\{\begin{aligned}
N_{1}(x, y)= & S(t) x_{0}+\int_{0}^{t} S(t-s) f^{1}(s, x(s), y(s)) d s \\
& +\sum_{l=1}^{\infty} \int_{0}^{t} S(t-s) \sigma_{l}^{1}(t, x(s), y(s)) d B_{l}^{H}(s) \\
& +\sum_{0<t_{k}<t} S\left(t-t_{k}\right) I_{k}\left(x\left(t_{k}\right)\right), \quad t \in[0, T] . \\
N_{2}(x, y)= & S(t) y_{0}+\int_{0}^{t} S(t-s) f^{2}(s, x(s), y(s)) d s \\
& +\sum_{l=1}^{\infty} \int_{0}^{t} S(t-s) \sigma_{l}^{2}(t, x(s), y(s)) d B_{l}^{H}(s) \\
& +\sum_{0<t_{k}<t} S\left(t-t_{k}\right) \bar{I}_{k}\left(y\left(t_{k}\right)\right), \quad t \in[0, T] .
\end{aligned}\right.
$$


Clearly, the fixed points of $N$ are solutions to (1.1). In order to apply Theorem 3.2, we first show that $N$ is completely continuous. The proof will be carried out in several steps.

Step 1. $N$ is continuous.

Let $\left(x_{n}, y_{n}\right)$ be a sequence such that $\left(x_{n}, y_{n}\right) \rightarrow(x, y) \in P C \times P C$ as $n \rightarrow \infty$, and observe that thanks to $\left(H_{3}\right)-\left(H_{6}\right)$ and $\left(H_{7}\right), I_{k}, \bar{I}_{k}, k=1,2, . ., m$, are continuous.

Then

$$
\begin{aligned}
& \left|N_{1}\left(x_{n}(t), y_{n}(t)\right)-N_{1}(x(t), y(t))\right|_{X}^{2} \\
& \leq 3\left|\sum_{l=1}^{\infty} \int_{0}^{t} S(t-s)\left(\sigma_{l}^{1}\left(s, x_{n}(s), y_{n}(s)\right) \sigma_{l}^{1}(s, x(s), y(s))\right) d B_{l}^{H}(s)\right|_{X}^{2} \\
& \quad+3\left|\int_{0}^{t} S(t-s)\left(f^{1}\left(s, x_{n}(s), y_{n}(s)\right)-f^{1}(s, x(s), y(s))\right) d s\right|_{X}^{2} \\
& \quad+3\left|\sum_{0<t_{k}<t} S\left(t-t_{k}\right) I_{k}\left(x_{n}\left(t_{k}\right)\right)-\sum_{0<t_{k}<t} S\left(t-t_{k}\right) I_{k}\left(x\left(t_{k}\right)\right)\right|_{X}^{2} .
\end{aligned}
$$

Thus, we deduce

$$
\begin{aligned}
E \mid & N_{1}\left(x_{n}(t), y_{n}(t)\right)-\left.N_{1}(x(t), y(t))\right|_{X} ^{2} \\
\leq & 3 M^{2} c_{2}(H) H(2 H-1) T^{2 H-1} \int_{0}^{t} E\left\|\sigma^{1}\left(s, x_{n}(s), y_{n}(s)\right)-\sigma^{1}(s, x(s), y(s))\right\|^{2} d s \\
& +3 M^{2} \int_{0}^{t} E\left|f^{1}\left(s, x_{n}(s), y_{n}(s)\right)-f^{1}(s, x(s), y(s))\right|_{X}^{2} d s \\
& +3 M^{2} \sum_{0<t_{k}<t} E\left|I_{k}\left(x_{n}\left(t_{k}\right)\right)-I_{k}\left(x\left(t_{k}\right)\right)\right|_{X}^{2} .
\end{aligned}
$$

By the Lebesgue dominated convergence theorem, we have

$$
\begin{aligned}
& \sup _{t \in J} E\left|N_{1}\left(x_{n}(t), y_{n}(t)\right)-N_{1}(x(t), y(t))\right|_{X}^{2} \\
& \quad \leq \bar{c}_{H} \int_{0}^{T} E\left\|\sigma^{1}\left(s, x_{n}(s), y_{n}(s)\right)-\sigma^{1}(s, x(s), y(s))\right\|^{2} d s \\
& \quad+3 M^{2} \int_{0}^{T} E\left|f^{1}\left(s, x_{n}(s), y_{n}(s)\right)-f^{1}(s, x(s), y(s))\right|_{X}^{2} d s \\
& \quad+3 M^{2} \sum_{k=1}^{m} E\left|I_{k}\left(x_{n}\left(t_{k}\right)\right)-I_{k}\left(x\left(t_{k}\right)\right)\right|_{X}^{2} \rightarrow 0 \text { as } n \rightarrow \infty
\end{aligned}
$$

where

$$
\bar{c}_{H}=3 M^{2} c_{2}(H) H(2 H-1) T^{2 H-1} .
$$


Similarly

$$
\begin{aligned}
\sup _{t \in J} E\left|N_{2}\left(x_{n}(t), y_{n}(t)\right)-N_{1}(x(t), y(t))\right|_{X}^{2} \\
\leq \bar{c}_{H} \int_{0}^{T} E\left\|\sigma^{2}\left(s, x_{n}(s), y_{n}(s)\right)-\sigma^{2}(s, x(s), y(s))\right\|^{2} d s \\
\quad+2 M^{2} \int_{0}^{T} E\left|f^{2}\left(s, x_{n}(s), y_{n}(s)\right)-f^{2}(s, x(s), y(s))\right|_{X}^{2} d s \\
\quad+3 M^{2} \sum_{k=1}^{m} E\left|\bar{I}_{k}\left(y_{n}\left(t_{k}\right)\right)-\bar{I}_{k}\left(y\left(t_{k}\right)\right)\right|_{X}^{2} \rightarrow 0 \text { as } n \rightarrow \infty .
\end{aligned}
$$

Therefore, $N$ is continuous.

Step 2. $N$ maps bounded sets into bounded sets in $P C \times P C$. Indeed, it is enough to show that for any $q>0$, there exists a positive constant $\kappa$ such that for each $(x, y) \in B_{q}=\left\{(x, y) \in P C \times P C:\|x\|_{P C} \leq q,\|y\| \leq q\right\}$, we have

$$
\|N(x, y)\|_{P C} \leq \kappa=\left(\kappa_{1}, \kappa_{2}\right)
$$

Then, for each $t \in J$ and thanks to Lemma 2.2,

$$
\begin{aligned}
E\left|N_{1}(x(t), y(t))\right|_{X}^{2} \leq & 4 M^{2} E\left|x_{0}\right|_{X}^{2}+4 M^{2}\left\|c_{1}\right\|_{L^{1}}+4 M^{2} c_{2}(H) H(2 H-1) T^{2 H-1}\left\|\bar{c}_{1}\right\|_{L^{1}} \\
& +4 M^{2}\left(\sum_{0<t_{k}<t}\left(d_{k} E|x|_{X}^{2}+\lambda_{k}\right)\right) \\
& +4 M^{2} \int_{0}^{t}\left(c_{2}(H) H(2 H-1) T^{2 H-1} \bar{\alpha}_{1}(s)+\bar{a}_{1}(s)\right) E|x(s)|_{X}^{2} d s \\
& +4 M^{2} \int_{0}^{t}\left(c_{2}(H) H(2 H-1) T^{2 H-1} \bar{\beta}_{1}(s)+\bar{b}_{1}(s)\right) E|y(s)|_{X}^{2} d s \\
\leq & 4 M^{2} E\left|x_{0}\right|_{X}^{2}+4 M^{2}\left\|c_{1}\right\|_{L^{1}}+4 M^{2} c_{2}(H) H(2 H-1) T^{2 H-1}\left\|\bar{c}_{1}\right\|_{L^{1}} \\
& +4 M^{2} \sum_{0<t_{k}<t} \lambda_{k} \\
& +4 M^{2} \int_{0}^{t}\left(c_{2}(H) H(2 H-1) T^{2 H-1} \bar{\alpha}_{1}(s)+\bar{a}_{1}(s)\right) q d s \\
& +4 M^{2} \int_{0}^{t}\left(c_{2}(H) H(2 H-1) T^{2 H-1} \bar{\beta}_{1}(s)+\bar{b}_{1}(s)\right) q d s \\
& +4 M^{2} \sum_{0<t_{k}<t} d_{k} q=\kappa_{1} .
\end{aligned}
$$


Similarly, we have

$$
\begin{aligned}
E\left|N_{2}(x, y)\right|_{X}^{2} \leq & 4 M^{2} E\left|y_{0}\right|_{X}^{2}+4 M^{2}\left\|c_{2}\right\|_{L^{1}}+4 M^{2} c_{2}(H) H(2 H-1) T^{2 H-1}\left\|\bar{c}_{2}\right\|_{L^{1}} \\
& +4 M^{2} \sum_{0<t_{k}<t} \bar{\lambda}_{k} \\
& +4 M^{2} \int_{0}^{t}\left(c_{2}(H) H(2 H-1) T^{2 H-1} \bar{\alpha}_{2}(s)+\bar{a}_{2}(s)\right) q d s \\
& +4 M^{2} \int_{0}^{t}\left(c_{2}(H) H(2 H-1) T^{2 H-1} \bar{\beta}_{2}(s)+\bar{b}_{2}(s)\right) q d s \\
& +4 M^{2} \sum_{0<t_{k}<t} \bar{d}_{k} q=\kappa_{2} .
\end{aligned}
$$

Step $3 N$ maps bounded sets into equicontinuous sets of $P C \times P C$.

Let $B_{q}$ be a bounded set in $P C \times P C$ as in Step 2. Let $\tau_{1}, \tau_{2} \in J, \tau_{1}<\tau_{2}$ and $u \in B_{q}$. Then, for $\mathrm{i}=1,2$, we obtain

$$
\begin{aligned}
& E\left|N_{i}\left(\tau_{2}, x\left(\tau_{2}\right), y\left(\tau_{2}\right)\right)-N_{i}\left(\tau_{1}, x\left(\tau_{1}\right), y\left(\tau_{1}\right)\right)\right|_{X}^{2} \\
& \leq \\
& +\left\|\left(S\left(\tau_{2}\right)-S\left(\tau_{1}\right)\right)\right\|^{2} E\left|x_{0}\right|_{X}^{2} \\
& +7 E\left|\int_{0}^{\tau_{1}}\left(S\left(\tau_{2}-s\right)-S\left(\tau_{1}-s\right)\right) f^{i}(s, x(s), y(s)) d s\right|_{X}^{2} \\
& +7 E\left|\int_{\tau_{1}}^{\tau_{2}} S\left(\tau_{2}-s\right) f^{i}(s, x(s), y(s)) d s\right|_{X}^{2} \\
& +7 E\left|\sum_{l=1}^{\infty} \int_{0}^{\tau_{1}}\left(S\left(\tau_{2}-s\right)-S\left(\tau_{1}-s\right)\right) \sigma_{l}^{i}(s, x(s), y(s)) d B_{l}^{H}(s)\right|_{X}^{2} \\
& +7 E\left|\sum_{l=1}^{\infty} \int_{\tau_{1}}^{\tau_{2}} S\left(\tau_{2}-s\right) \sigma_{l}^{i}(s, x(s), y(s)) d B_{l}^{H}(s)\right|_{X}^{2} \\
& +7 E\left|\sum_{0<t_{k}<\tau_{2}}\left(S\left(\tau_{1}-t_{k}\right)-S\left(\tau_{2}-t_{k}\right)\right) I_{k}\left(x\left(t_{k}\right)\right)\right|_{X}^{2} \\
& +7 E\left|\sum_{\tau_{1}<t_{k}<\tau_{2}}\left(S\left(\tau_{2}-t_{k}\right)\right) I_{k}\left(x\left(t_{k}\right)\right)\right|_{X}^{2} .
\end{aligned}
$$


From Lemma 2.2 we deduce

$$
\begin{aligned}
& E\left|N_{i}\left(\tau_{2}, x\left(\tau_{2}\right), y\left(\tau_{2}\right)\right)-N_{i}\left(\tau_{1}, x\left(\tau_{1}\right), y\left(\tau_{1}\right)\right)\right|_{X}^{2} \\
& \leq\left.7 \|\left(S\left(\tau_{2}-\tau_{1}\right)-I d\right)\right) \|^{2} E\left|x_{0}\right|_{X}^{2} \\
&+7 \tau_{2} \int_{0}^{\tau_{1}} \|\left(S\left(\tau_{2}-s\right)-S\left(\tau_{1}-s\right) \|^{2} E\left|f^{i}(s, x(s), y(s))\right|_{X}^{2} d s\right. \\
&+7\left(\tau_{2}-\tau_{1}\right) \int_{\tau_{1}}^{\tau_{2}}\left\|S\left(\tau_{2}-s\right)\right\|^{2} E\left|f^{i}(s, x(s), y(s))\right|_{X}^{2} d s \\
&+7 c_{2}(H) H(2 H-1) \tau_{2}^{2 H-1} \int_{0}^{\tau_{1}}\left\|S\left(\tau_{2}-s\right)-S\left(\tau_{1}-s\right)\right\|^{2} E\left\|\sigma^{i}(s, x(s), y(s))\right\|^{2} d s \\
&+7 c_{2}(H) H(2 H-1)\left|\tau_{2}-\tau_{1}\right|^{2 H-1} \int_{\tau_{1}}^{\tau_{2}} \mid\left(S\left(\tau_{1}-s\right)\left\|^{2} E\right\| \sigma^{i}(s, x(s), y(s)) \|^{2} d s\right. \\
&\left.+7 \sum_{0<t_{k}<\tau_{2}} \| S\left(\tau_{2}-\tau_{1}\right)-I_{d}\right) \|^{2} E\left|I_{k}\left(x\left(t_{k}\right)\right)\right|_{X}^{2} \\
&\left.+7 \sum_{\tau_{1}<t_{k}<\tau_{2}} \| S\left(\tau_{2}-t_{k}\right)\right) \|^{2} E\left|I_{k}\left(x\left(t_{k}\right)\right)\right|_{X}^{2} .
\end{aligned}
$$

From $\left(H_{3}\right)-\left(H_{4}\right)$ and $\left(H_{6}\right)$ we have

$$
\begin{aligned}
E \mid N_{i} & \left(\tau_{2}, x\left(\tau_{2}\right), y\left(\tau_{2}\right)\right)-\left.N_{i}\left(\tau_{1}, x\left(\tau_{1}\right), y\left(\tau_{1}\right)\right)\right|_{X} ^{2} \\
\leq & 7\left\|S\left(\tau_{2}-\tau_{1}\right)-I d\right\|^{2} E\left|x_{0}\right|_{X}^{2} \\
& +7 \tau_{2} \int_{0}^{\tau_{2}}\left\|S\left(\tau_{2}-s\right)-S\left(\tau_{1}-s\right)\right\|^{2}\left(\bar{a}_{i}(s) E|x(s)|_{X}^{2}+\bar{b}_{i}(s) E|y(s)|_{X}^{2}+c_{i}(s)\right) d s \\
& +7\left(\tau_{2}-\tau_{1}\right) \int_{\tau_{1}}^{\tau_{2}}\left\|S\left(\tau_{1}-s\right)\right\|^{2}\left(\bar{a}_{i}(s) E|x(s)|_{X}^{2}+\bar{b}_{i}(s) E|y(s)|_{X}^{2}+c_{i}(s)\right) d s \\
& +7 c_{2}(H) H(2 H-1) \tau_{2}^{2 H-1} \times \\
& \times \int_{0}^{\tau_{2}}\left\|S\left(\tau_{2}-s\right)-S\left(\tau_{1}-s\right)\right\|^{2}\left(\bar{\alpha}_{i}(s) E|x(s)|_{X}^{2}+\bar{\beta}_{i}(s) E|y(s)|_{X}^{2}+\bar{c}_{i}(s)\right) d s \\
& +7 c_{2}(H) H(2 H-1)\left(\tau_{2}-\tau_{1}\right)^{2 H-1} \times \\
& \times \int_{\tau_{1}}^{\tau_{2}} \|\left(S\left(\tau_{1}-s\right) \|^{2}\left(\bar{\alpha}_{i}(s) E|x(s)|_{X}^{2}+\bar{\beta}_{i}(s) E|y(s)|_{X}^{2}+\bar{c}_{i}(s)\right) d s\right. \\
& \left.+7 \sum_{0<t_{k}<\tau_{2}} \| S\left(\tau_{2}-\tau_{1}\right)-I_{d}\right) \|^{2}\left(d_{k} E\left|x\left(t_{k}\right)\right|_{X}^{2}+\lambda_{k}\right) \\
& +7 \sum_{\tau_{1}<t_{k}<\tau_{2}}\left\|S\left(\tau_{2}-t_{k}\right)\right\|^{2}\left(d_{k} E\left|x\left(t_{k}\right)\right|_{X}^{2}+\lambda_{k}\right) .
\end{aligned}
$$

Now, it is straightforward to see that the right-hand side tends to zero as $\tau_{2}-\tau_{1} \rightarrow 0$ since the compactness of $S(t)$ for $t>0$ implies the continuity in the uniform operator topology (see [28]). This proves the equicontinuity. 
Step $4\left(N\left(B_{q}\right)(t)\right.$ is precompact in $X \times X$.

As a consequence of Steps 2 to 3, and at light of the Arzelá-Ascoli theorem, it is sufficient to show that $N$ maps $B_{q}$ into a precompact set in $X \times X$. Let $0<t<b$ be fixed and let $\epsilon$ be a real number satisfying $0<\epsilon<t$. For $(x, y) \in B_{q}$ we define

$$
\begin{aligned}
N_{i}^{\epsilon}(x, y)= & S(t) z_{0}^{i}+\int_{0}^{t-\epsilon} S(t-s) f^{i}(s, x(s), y(s)) d s \\
& +\sum_{l=1}^{\infty} \int_{0}^{t-\epsilon} S(t-s) \sigma_{l}^{i}(t, x(s), y(s)) d B_{l}^{H}(s) \\
& +\sum_{0<t_{k}<t-\epsilon} S\left(t-t_{k}\right) I_{k}^{i}\left(z\left(t_{k}\right)\right) \\
= & S(\epsilon) S(t-\epsilon) z_{0}^{i}+S(\epsilon) \int_{0}^{t-\epsilon} S(t-s-\epsilon) f^{i}(s, x(s), y(s)) d s \\
& +S(\epsilon) \sum_{l=1}^{\infty} \int_{0}^{t-\epsilon} S(t-s-\epsilon) \sigma_{l}^{i}(t, x(s), y(s)) d B_{l}^{H}(s) \\
& +\sum_{0<t_{k}<t-\epsilon} S\left(t-t_{k}\right) I_{k}^{i}\left(z^{i}\left(t_{k}\right)\right) .
\end{aligned}
$$

Since $S(t)$ is a compact operator, the set

$$
H_{\epsilon}=\left\{N^{\epsilon}(x, y)(t)=\left(N_{1}^{\epsilon}(x, y)(t), N_{2}^{\epsilon}(x, y)(t)\right)(x, y) \in B_{q}\right\}
$$

is precompact in $X \times X$ for every $\epsilon$ such that $0<\epsilon<t$ Moreover, for every $(x, y) \in B_{q}$, and $i=1,2$ we have

$$
\begin{aligned}
E\left\|N_{i}(x, y)-N_{i}^{\epsilon}(x, y)\right\|^{2} \leq & 3 \int_{t-\epsilon}^{t}\left(\bar{a}_{i}(s) q+\bar{b}_{i}(s) q+c_{i}(s)\right) d s \\
& +3 M^{2} c_{2}(H) H(2 H-1) T^{2 H-1} \int_{t-\epsilon}^{t}\left(\bar{\alpha}_{i}(s) q+\bar{\beta}_{i}(s) q+\bar{c}_{i}(s)\right) d s \\
& +3 M^{2} \sum_{t-\epsilon<t_{k}<t}\left(d_{k} q+\lambda_{k}\right) .
\end{aligned}
$$

Therefore, there are precompact sets arbitrarily close to the set $H_{\epsilon}=\left\{N^{\epsilon}(x, y)(t)=\right.$ $\left.\left(N_{1}^{\epsilon}(x, y)(t), N_{2}^{\epsilon}(x, y)(t)\right), \quad(x, y) \in B_{q}\right\}$.

Hence, the set $H=\left\{N(x, y)(t)=\left(N_{1}(x, y)(t), N_{2}(x, y)(t)\right), \quad(x, y) \in B_{q}\right\}$ is precompact in $X \times X$ and the right-hand side tends to 0 uniformly in $t$ as $\epsilon \rightarrow 0^{+}$. Hence we can conclude the relative compactness of $N\left(B_{q}\right)(t)$ for $t \geq 0$. By the Arzelá-Ascoli theorem, we conclude that $N: P C \times P C \rightarrow P C \times P C$ is a completely continuous operator.

Step 5 A priori bounds on solutions.

The set

$$
U=\left\{(x, y) \in P C \times P C: \quad x=\lambda N_{1}(x, y) \text { and } y=\lambda N_{2}(x, y) \text { for some } 0<\lambda<1\right\}
$$


is bounded.

Let $(x, y) \in P C \times P C$ be a solution of the abstract nonlinear equation $x=\lambda N_{1}(x, y)$ and $y=\lambda N_{2}(x, y)$ for some $0<\lambda<1$. Thus, for $t \in\left[0, t_{1}\right]$,

$$
\begin{aligned}
& E|x(t)|_{X}^{2} \leq 3 M^{2} E\left|x_{0}\right|_{X}^{2}+3 M^{2} \int_{0}^{t}\left(\bar{a}_{1}(s) E|x(s)|_{X}^{2}+\bar{b}_{1}(s) E|y(s)|_{X}^{2}+c_{1}(s)\right) d s \\
&+3 M^{2} c_{2}(H) H(2 H-1) t_{1}^{2 H-1} \int_{0}^{t}\left(\bar{\alpha}_{1}(s) E|x(s)|_{X}^{2}+\bar{\beta}_{1}(s) E|y(s)|_{X}^{2}+\bar{c}_{1}(s)\right) d s \\
& \leq 3 M^{2} E\left|x_{0}\right|^{2}+3 M^{2}\left\|c_{1}\right\|_{L^{1}}+3 M^{2} c_{2}(H) H(2 H-1) t_{1}^{2 H-1}\left\|\bar{c}_{1}\right\|_{L^{1}} \\
&+3 M^{2} \int_{0}^{t}\left(c_{2}(H) H(2 H-1)^{2} t_{1}^{2 H-1} \bar{\alpha}_{1}(s)+\bar{a}_{1}(s)\right) E|x(s)|_{X}^{2} d s \\
&+3 M^{2} \int_{0}^{t}\left(c_{2}(H) H(2 H-1)^{2} t_{1}^{2 H-1} \bar{\beta}_{1}(s)+\bar{b}_{1}(s)\right) E|y(s)|_{X}^{2} d s \\
& \\
& E|x(t)|_{X}^{2} \leq A_{1}+\int_{0}^{t} B_{1}(s) E|x(s)|_{X}^{2} d s+\int_{0}^{t} C_{1}(s) E|y(s)|_{X}^{2} d s
\end{aligned}
$$

and similarly

$$
E|y(t)|_{X}^{2} \leq A_{2}+\int_{0}^{t} B_{2}(s) E|x(s)|_{X}^{2} d s+\int_{0}^{t} C_{2}(s) E|y(s)|_{X}^{2},
$$

where for each $j=1,2$

$$
\begin{aligned}
& A_{j}=3 M^{2} E\left|z_{0}^{j}\right|^{2}+3 M^{2}\left\|c_{j}\right\|_{L^{1}}+3 M^{2} c_{2}(H) H(2 H-1) t_{1}^{2 H-1}\left\|\bar{c}_{j}\right\|_{L^{1}} \\
& \left.B_{j}(s)=3 M^{2}\left(c_{2}(H) H(2 H-1)^{2} t_{1}^{2 H-1} \bar{\alpha}_{i}(s)+\bar{a}_{i}(s)\right)\right) \quad s \in\left[0, t_{1}\right]
\end{aligned}
$$

and

$$
C_{j}(s)=3 M^{2}\left(c_{2}(H) H(2 H-1)^{2} t_{1}^{2 H-1} \bar{\beta}_{j}(s)+\bar{b}_{j}(s)\right),
$$

and $z_{0}^{1}=x_{0}$ and $z_{0}^{2}=y_{0}$.

From these previous expressions we easily deduce

$$
\sup _{r \in[0, t]}\left(E|y(r)|_{X}^{2}+E|x(r)|_{X}^{2}\right) \leq K_{1}+\int_{0}^{t} K_{2}(s) \sup _{\eta \in[0, s]}\left(E|x(\eta)|_{X}^{2}+E|y(\eta)|_{X}^{2}\right) d s
$$

where

$$
K_{1}=A_{1}+A_{2}, \quad K_{2}(s)=\max \left\{B_{1}(s)+B_{2}(s), C_{1}(s)+C_{2}(s)\right\} .
$$

Using now the Gronwall inequality

$$
\sup _{r \in[0, t]}\left(E|y(r)|_{X}^{2}+E|x(r)|_{X}^{2}\right) \leq K_{1} \exp \left(\int_{0}^{t_{1}} K_{2}(s) d s\right)=\bar{M}_{0}
$$


whence

$$
\sup _{t \in\left[0, t_{1}\right]} E|x(t)|_{X}^{2} \leq \bar{M}_{0}, \text { and } \sup _{t \in\left[0, t_{1}\right]} E|y(t)|_{X}^{2} \leq \bar{M}_{0}
$$

where $\bar{M}_{0}$ depends only on $t_{1}$. For $t \in\left(t_{1}, t_{2}\right]$, we have

$$
\begin{aligned}
x(t)= & S\left(t-t_{1}\right)\left[x\left(t_{1}\right)+I_{1}\left(x_{1}\left(t_{1}^{-}\right)\right)\right]+\int_{t_{1}}^{t} S(t-s) f^{1}(s, x(s), y(s)) d s \\
& +\sum_{l=1}^{\infty} \int_{t_{1}}^{t} S(t-s) \sigma_{l}^{1}(t, x(s), y(s)) d B_{l}^{H}(s) .
\end{aligned}
$$

Then, by a similar argument we obtain

$$
\begin{aligned}
E|x(t)|_{X}^{2}= & E \mid S\left(t-t_{1}\right)\left[x_{1}\left(t_{1}\right)+I_{1}\left(x_{1}\left(t_{1}^{-}\right)\right)\right]+\int_{t_{1}}^{t} S(t-s) f^{1}(s, x(s), y(s)) d s \\
& +\left.\sum_{l=1}^{\infty} \int_{t_{1}}^{t} S(t-s) \sigma_{l}^{1}(t, x(s), y(s)) d B_{l}^{H}(s)\right|_{X} ^{2} \\
\leq & \left\|S\left(t-t_{1}\right)\right\|^{2}\left(E\left|x_{1}\left(t_{1}\right)\right|_{X}^{2}+E\left|I_{1}\left(x_{1}\left(t_{1}^{-}\right)\right)\right|_{X}^{2}\right)+E\left|\int_{t_{1}}^{t} S(t-s) f^{1}(s, x(s), y(s)) d s\right|_{X}^{2} \\
& +E\left|\sum_{l=1}^{\infty} \int_{t_{1}}^{t} S(t-s) \sigma_{l}^{1}(t, x(s), y(s)) d B_{l}^{H}(s)\right|_{X}^{2} \\
\leq & 4 M^{2}\left(\bar{M}^{2}+d_{1} E \mid x\left(\left.t_{1}\right|_{X} ^{2}+\lambda\right)+4 M^{2} \int_{t_{1}}^{t}\left(\bar{a}_{1}(s) E|x(s)|_{X}^{2}+\bar{b}_{1}(s) E|y(s)|_{X}^{2}+c_{1}(s)\right) d s\right. \\
& +4 M^{2} c_{2}(H) H(2 H-1) t_{2}^{2 H-1} \int_{t_{1}}^{t}\left(\bar{\alpha}_{1}(s) E|x(s)|_{X}^{2}+\bar{\beta}_{1}(s) E|y(s)|_{X}^{2}+\bar{c}_{1}(s)\right) d s \\
\leq & A_{3}+\int_{t_{1}}^{t} B_{3}(s) E|x(s)|_{X}^{2} d s+\int_{t_{1}}^{t} C_{3}(s) E|y(s)|_{X}^{2} d s .
\end{aligned}
$$

Then we deduce

$$
E|y(t)|_{X}^{2} \leq A_{4}+\int_{t_{1}}^{t} B_{4}(s) E|x(s)|_{X}^{2} d s+\int_{t_{1}}^{t} C_{4}(s) E|y(s)|_{X}^{2},
$$

where

$$
A_{3}=4 M^{2}\left(\bar{M}^{2}+\lambda\right)+4 M^{2}\left\|c_{1}\right\|_{L^{1}}+4 M^{2} c_{2}(H) H(2 H-1) t_{2}^{2 H-1}\left\|\bar{c}_{1}\right\|_{L^{1}}
$$

and

$$
A_{4}=4 M^{2}\left(\bar{M}^{2}+\bar{\lambda}\right)+4 M^{2}\left\|c_{2}\right\|_{L^{1}}+4 M^{2} c_{2}(H) H(2 H-1) t_{2}^{2 H-1}\left\|\bar{c}_{2}\right\|_{L^{1}},
$$


and

$$
\begin{gathered}
B_{3}(s)=4 M^{2} d_{1}+4 M^{2}\left(c_{2}(H) H(2 H-1)^{2} t_{2}^{2 H-1} \bar{\alpha}_{1}(s)+\bar{a}_{1}(s)\right) \\
B_{4}(s)=4 M^{2}+\left(c_{2}(H) H(2 H-1)^{2} t_{2}^{2 H-1} \bar{\alpha}_{2}(s)+\bar{a}_{2}(s)\right), \quad s \in\left(t_{1}, t_{2}\right]
\end{gathered}
$$

and

$$
\begin{gathered}
C_{3}(s)=4 M^{2}\left(c_{2}(H) H(2 H-1)^{2} t_{2}^{2 H-1} \bar{\beta}_{1}(s)+\bar{b}_{1}(s)\right), \quad s \in\left(t_{1}, t_{2}\right], \\
C_{4}(s)=4 M^{2} \bar{d}_{1}+4 M^{2}\left(c_{2}(H) H(2 H-1)^{2} t_{2}^{2 H-1} \bar{\beta}_{1}(s)+\bar{b}_{2}(s)\right), \quad s \in\left(t_{1}, t_{2}\right] .
\end{gathered}
$$

Combining $E|x(t)|_{X}^{2}$ and $E|y(t)|_{X}^{2}$,

$$
\sup _{r \in\left[t_{1}, t\right]}\left(E|y(r)|_{X}^{2}+E|x(r)|_{X}^{2}\right) \leq K_{3}+\int_{t_{1}}^{t} K_{4}(s) \sup _{\eta \in\left[t_{1}, s\right]}\left(E|x(\eta)|_{X}^{2}+E|y(\eta)|_{X}^{2}\right) d s
$$

where

$$
K_{3}=A_{3}+A_{4}, \quad K_{4}(s)=\max \left\{B_{3}(s)+B_{4}(s), C_{3}(s)+C_{4}(s)\right\} .
$$

Using once more the Gronwall inequality,

$$
\sup _{t \in\left(t_{1}, t_{2}\right]}\left(E|y(t)|_{X}^{2}+E|x(t)|_{X}^{2}\right) \leq K_{3} \exp \left(\int_{t_{1}}^{t_{2}} K_{4}(s) d s\right)=\bar{M}_{1} .
$$

Consequently, there exists a constant $\bar{M}_{1}$ which only depends on $t_{1}, t_{2}$ such that

$$
\sup _{t \in\left(t_{1}, t_{2}\right]} E|x(t)|_{X}^{2} \leq \bar{M}_{1} \text {, and } \sup _{t \in\left(t_{1}, t_{2}\right]} E|y(t)|_{X}^{2} \leq \bar{M}_{1}
$$

If we iterate this procedure in every interval $\left(t_{i}, t_{i+1}\right]$ we can prove that there exists a positive constant $\bar{M}_{i}$, which only depends on $t_{i}, t_{i+1}$, such that

$$
\sup _{t \in\left(t_{i}, t_{i+1}\right]} E|x(t)|_{X}^{2} \leq \bar{M}_{i}, \text { and } \sup _{t \in\left(t_{i}, t_{i+1}\right]} E|y(t)|_{X}^{2} \leq \bar{M}_{i}, \quad i=0,1,2, \ldots, m-1 .
$$

Set

where

$$
U=\left\{(x, y) \in P C \times P C: \quad\|x\|_{P C}<M^{*}, \quad\|y\|_{P C}<M^{*}\right\}
$$

$$
M^{*}=\sup \left\{\bar{M}_{i}: \quad i=0,1,2, \ldots, m-1\right\} .
$$

Then, $U$ is an open subset of $P C \times P C$ and it is straightforward to see that there is no $(x, y) \in \partial U$ such that $x=\lambda N_{1}(x, y)$ and $y=\lambda N_{2}(x, y)$ for some $0<\lambda<1$. By Theorem 3.2, $N$ possesses at least one fixed point $(x, y)$.

Remark 4.1. We can replace conditions $\left(H_{3}\right)$ and $\left(H_{5}\right)$ by the following hypotheses $(\widetilde{H})$ there exist integrable functions $\eta:[0, T] \longrightarrow \mathbb{R}^{+}$and $\psi:[0, \infty) \longrightarrow(0, \infty)$ which are continuous and nondecreasing such that, for all $t \in[0, T]$ and $x, y \in X$,

$$
E\left\|f^{i}(t, x, y)\right\|^{2} \leq \eta(t) \psi\left(E\|x\|^{2}+E\|y\|^{2}\right), \quad E\left\|\sigma^{i}(t, x, y)\right\|^{2} \leq \eta(t) \psi\left(E|x|^{2}+E|y|^{2}\right),
$$

where

$$
\sigma^{i}(t, x, y)=\sum_{l=1}^{\infty} \sigma_{l}^{i}(t, x, y), \quad i=1,2
$$




\section{$5 \quad$ Weak solutions}

In this section we prove that mild solutions to system (1.1) are also weak solutions. First, we recall the definition of weak solution according to Da Prato and Zabczyk [11]. To shorten the notation, we will use $\langle.,$.$\rangle instead of (.,$.$) below since no confusion is$ possible.

Definition 5.1. An $X$-valued stochastic process $u(t)=(x(t), y(t)), t \in[0, T]$ is called a weak solution of (1.1) if for each $\varphi \in D\left(A^{*}\right)$

$$
\left\{\begin{aligned}
\langle x(t), \varphi\rangle & =\left\langle x_{0}, \varphi\right\rangle+\int_{0}^{t}\left\langle x(s), A^{*} \varphi\right\rangle d \tau+\int_{0}^{t}\left\langle f^{1}(\tau, x(\tau), y(\tau)), \varphi\right\rangle d \tau \\
& +\sum_{l=1}^{\infty} \int_{0}^{t}\left\langle\sigma_{l}^{1}(\tau, x(\tau), y(\tau)), \varphi\right\rangle d B_{l}^{H}(\tau) \\
& +\sum_{0<t_{k}<t}\left\langle I_{k}\left(x\left(t_{k}\right)\right), \varphi\right\rangle, \quad t \in J \\
\langle y(t), \varphi\rangle= & \left\langle y_{0}, \varphi\right\rangle+\int_{0}^{t}\left\langle y(s), A^{*} \varphi\right\rangle d \tau+\int_{0}^{t}\left\langle f^{2}(\tau, x(\tau), y(\tau)), \varphi\right\rangle d \tau \\
& +\sum_{l=1}^{\infty} \int_{0}^{t}\left\langle\sigma_{l}^{2}(\tau, x(\tau), y(\tau)), \varphi\right\rangle d B_{l}^{H}(\tau) \\
& +\sum_{0<t_{k}<t}\left\langle\bar{I}_{k}\left(y\left(t_{k}\right)\right), \varphi\right\rangle, \quad t \in J .
\end{aligned}\right.
$$

Theorem 5.1. Assume conditions $\left(H_{3}\right)-\left(H_{6}\right)$ hold. The mild solution of (1.1) is also a weak solution.

Proof. Let $(x(t), y(t))$ be a mild solution to (1.1). Then, for each $\varphi \in D\left(A^{*}\right)$ and 
$t \in\left[0, t_{1}\right]$, we obtain

$$
\begin{aligned}
& E \mid \int_{0}^{t}\left\langle x(s), A^{*} \varphi\right\rangle d s-\int_{0}^{t}\left\langle S(s) x_{0}, A^{*} \varphi\right\rangle d s-\int_{0}^{t} \int_{0}^{s}\left\langle S(s-\tau) f^{1}(\tau, x(\tau), y(\tau)), A^{*} \varphi\right\rangle d \tau d s \\
& \quad-\sum_{l=1}^{\infty} \int_{0}^{t} \int_{0}^{s}\left\langle S(s-\tau) \sigma_{l}^{1}(\tau, x(\tau), y(\tau)), A^{*} \varphi\right\rangle d B_{l}^{H}(\tau) d s \mid \\
& \leq \int_{0}^{t} E \mid\left\langle x(s), A^{*} \varphi\right\rangle-\left\langle S(t) x_{0}, A^{*} \varphi\right\rangle-\int_{0}^{s}\left\langle S(s-\tau) f^{1}(\tau, x(\tau), y(\tau)), A^{*} \varphi\right\rangle d \tau \\
& \quad-\sum_{l=1}^{\infty} \int_{0}^{s}\left\langle S(s-\tau) \sigma_{l}^{1}(\tau, x(\tau), y(\tau)), A^{*} \varphi\right\rangle d B_{l}^{H}(\tau) d s \mid d s \\
& =\int_{0}^{t} E \mid\left\langle x(s)-S(s) x_{0}-\int_{0}^{s} S(s-\tau) f^{1}(\tau, x(\tau), y(\tau)) d \tau\right. \\
& \left.\left.\quad-\sum_{l=1}^{\infty} \int_{0}^{s} S(s-\tau) \sigma_{l}^{1}(\tau, x(\tau), y(\tau)) d B_{l}^{H}(\tau) d s\right) d s, A^{*} \varphi\right\rangle \mid d s \\
& =0 .
\end{aligned}
$$

Thus,

$$
\begin{aligned}
\int_{0}^{t}\left\langle x(s), A^{*} \varphi\right\rangle d s= & \int_{0}^{t}\left\langle S(t) x_{0}, A^{*} \varphi\right\rangle d s+\int_{0}^{t} \int_{0}^{s}\left\langle S(s-\tau) f^{1}(\tau, x(\tau), y(\tau)), A^{*} \varphi\right\rangle d \tau d s \\
& +\sum_{l=1}^{\infty} \int_{0}^{t} \int_{0}^{s}\left\langle S(s-\tau) \sigma_{l}^{1}(\tau, x(\tau), y(\tau)), A^{*} \varphi\right\rangle d B_{l}^{H}(\tau) d s \\
= & \sum_{j=1}^{3} \widetilde{P}_{j}
\end{aligned}
$$

Similarly,

$$
\begin{aligned}
\int_{0}^{t}\left\langle y(s), A^{*} \varphi\right\rangle d s= & \int_{0}^{t}\left\langle S(t) y_{0}, A^{*} \varphi\right\rangle d s+\int_{0}^{t} \int_{0}^{s}\left\langle S(s-\tau) f^{2}(\tau, x(\tau), y(\tau)), A^{*} \varphi\right\rangle d \tau d s \\
& +\sum_{l=1}^{\infty} \int_{0}^{t} \int_{0}^{s}\left\langle S(s-\tau) \sigma_{l}^{2}(\tau, x(\tau), y(\tau)), A^{*} \varphi\right\rangle d B_{l}^{H}(\tau) d s \\
= & \sum_{j=1}^{3} \widehat{P}_{j} .
\end{aligned}
$$

Now for $\varphi \in D\left(A^{*}\right)$ and $t \in\left[0, t_{1}\right]$ we use the fact that $\frac{d}{d t} S^{*}(t) \varphi=S^{*}(t) A^{*} \varphi$, which 
yields

$$
\begin{aligned}
\widetilde{P}_{1}=\int_{0}^{t}\left\langle S(s) x_{0}, A^{*} \varphi\right\rangle d s & =\int_{0}^{t}\left\langle x_{0}, S^{*}(s) A^{*} \varphi\right\rangle d s \\
& =\int_{0}^{t}\left\langle x_{0}, \frac{d}{d s} S^{*}(s) \varphi\right\rangle d s \\
& =\left\langle S(t) x_{0}-x_{0}, \varphi\right\rangle,
\end{aligned}
$$

and analogously,

$$
\widehat{P}_{1}=\int_{0}^{t}\left\langle S(s) y_{0}, A^{*} \varphi\right\rangle=\left\langle S(t) y_{0}-y_{0}, \varphi\right\rangle .
$$

In addition, using Fubini's Theorem for $t \in\left[0, t_{1}\right]$,

$$
\begin{aligned}
\widetilde{P}_{2} & =\int_{0}^{t} \int_{0}^{s}\left\langle S(s-\tau) f^{1}(\tau, x(\tau), y(\tau)), A^{*} \varphi\right\rangle d \tau d s \\
& =\int_{0}^{t} \int_{\tau}^{t}\left\langle 1_{(0, s]} f^{1}(\tau, x(\tau), y(\tau)), S^{*}(s-\tau) A^{*} \varphi\right\rangle d \tau d s \\
& =\int_{0}^{t}\left\langle S(t-\tau) f^{1}(\tau, x(\tau), y(\tau))-f^{1}(\tau, x(\tau), y(\tau)), \varphi\right\rangle d \tau \\
& \left.=\int_{0}^{t}\left\langle S(t-\tau) f^{1}(\tau, x(\tau), y(\tau)), y(\tau)\right), \varphi\right\rangle d \tau-\int_{0}^{t}\left\langle f^{1}(\tau, x(\tau), y(\tau)), \varphi\right\rangle d \tau
\end{aligned}
$$

and

$$
\begin{aligned}
\widehat{P}_{2} & =\int_{0}^{t} \int_{0}^{s}\left\langle S(s-\tau) f^{2}(\tau, x(\tau), y(\tau)), A^{*} \varphi\right\rangle d \tau d s \\
& \left.=\int_{0}^{t}\left\langle S(t-\tau) f^{2}(\tau, x(\tau), y(\tau)), y(\tau)\right), \varphi\right\rangle d \tau-\int_{0}^{t}\left\langle f^{2}(\tau, x(\tau), y(\tau)), \varphi\right\rangle d \tau
\end{aligned}
$$

As for the estimates of the third terms we have for $t \in\left[0, t_{1}\right]$

$$
\begin{aligned}
\widetilde{P}_{3} & =\sum_{l=1}^{\infty} \int_{0}^{t} \int_{0}^{s}\left\langle S(s-\tau) \sigma_{l}^{1}(\tau, x(\tau), y(\tau)), A^{*} \varphi\right\rangle d B_{l}^{H}(\tau) d s \\
& =\sum_{l=1}^{\infty} \int_{0}^{t} \int_{\tau}^{t}\left\langle 1_{(0, s]} \sigma_{l}^{1}(\tau, x(\tau), y(\tau)), S^{*}(s-\tau) A^{*} \varphi\right\rangle d B_{l}^{H}(\tau) d s \\
& =\sum_{l=1}^{\infty} \int_{0}^{t}\left\langle S(t-\tau) \sigma_{l}^{1}(\tau, x(\tau), y(\tau)), \varphi\right\rangle d B_{l}^{H}(\tau)-\sum_{l=1}^{\infty} \int_{0}^{t}\left\langle\sigma_{l}^{1}(\tau, x(\tau), y(\tau)), \varphi\right\rangle d B_{l}^{H}(\tau),
\end{aligned}
$$

and

$$
\begin{aligned}
\widehat{P}_{3} & =\sum_{l=1}^{\infty} \int_{0}^{t} \int_{0}^{s}\left\langle S(s-\tau) \sigma_{l}^{2}(\tau, x(\tau), y(\tau)), A^{*} \varphi\right\rangle d B_{l}^{H}(\tau) d s \\
& =\sum_{l=1}^{\infty} \int_{0}^{t}\left\langle S(t-\tau) \sigma_{l}^{2}(\tau, x(\tau), y(\tau)), \varphi\right\rangle d B_{l}^{H}(\tau)-\sum_{l=1}^{\infty} \int_{0}^{t}\left\langle\sigma_{l}^{2}(\tau, x(\tau), y(\tau)), \varphi\right\rangle d B_{l}^{H}(\tau) .
\end{aligned}
$$


Now, taking into account all the previous estimates we can write

$$
\begin{aligned}
\int_{0}^{t}\left\langle x(s), A^{*} \varphi\right\rangle d s= & \int_{0}^{t}\langle A x(s), \varphi\rangle d s=\left\langle S(t) x_{0}-x_{0}, \varphi\right\rangle \\
& \left.+\int_{0}^{t}\left\langle S(t-\tau) f^{1}(\tau, x(\tau), y(\tau)), y(\tau)\right), \varphi\right\rangle d \tau \\
& -\int_{0}^{t}\left\langle f^{1}(\tau, x(\tau), y(\tau)), \varphi\right\rangle d \tau \\
& +\sum_{l=1}^{\infty} \int_{0}^{t}\left\langle S(t-\tau) \sigma_{l}^{1}(\tau, x(\tau), y(\tau)), \varphi\right\rangle d B_{l}^{H}(\tau) \\
& -\sum_{l=1}^{\infty} \int_{0}^{t}\left\langle\sigma_{l}^{1}(\tau, x(\tau), y(\tau)), \varphi\right\rangle d B_{l}^{H}(\tau) \\
= & \left\langle x(t)-x_{0}, \varphi\right\rangle-\int_{0}^{t}\left\langle f^{1}(\tau, x(\tau), y(\tau)), \varphi\right\rangle d \tau \\
& -\sum_{l=1}^{\infty} \int_{0}^{t}\left\langle\sigma_{l}^{1}(\tau, x(\tau), y(\tau)), \varphi\right\rangle d B_{l}^{H}(\tau) .
\end{aligned}
$$

Finally,

$$
\begin{aligned}
\langle x(t), \varphi\rangle & =\left\langle x_{0}, \varphi\right\rangle+\int_{0}^{t}\left\langle x(s), A^{*} \varphi\right\rangle d \tau+\int_{0}^{t}\left\langle f^{1}(\tau, x(\tau), y(\tau)), \varphi\right\rangle d \tau \\
& +\sum_{l=1}^{\infty} \int_{0}^{t}\left\langle\sigma_{l}^{1}(\tau, x(\tau), y(\tau)), \varphi\right\rangle d B_{l}^{H}(\tau),
\end{aligned}
$$

and similar computations for $y$ for $t \in\left[0, t_{1}\right]$ imply

$$
\begin{aligned}
\langle y(t), \varphi\rangle & =\left\langle y_{0}, \varphi\right\rangle+\int_{0}^{t}\left\langle y(s), A^{*} \varphi\right\rangle d \tau+\int_{0}^{t}\left\langle f^{2}(\tau, x(\tau), y(\tau)), \varphi\right\rangle d \tau \\
& +\sum_{l=1}^{\infty} \int_{0}^{t}\left\langle\sigma_{l}^{2}(\tau, x(\tau), y(\tau)), \varphi\right\rangle d B_{l}^{H}(\tau) .
\end{aligned}
$$

Therefore the mild solution is also a weak solution in the interval $\left[0, t_{1}\right]$.

We repeat this scheme in every subinterval $\left.\left(t_{m-1}, t_{m}\right]\right)$ until we reach the points $t \in\left(t_{m}, T\right]$. As the arguments are the same as in the previous case, we prefer to omit the details.

\section{Exponential stability}

As in this section we are interested in the exponential decay to zero in mean square of the mild solutions to (1.1), we will assume that solutions are defined globally in time. 


$$
\begin{aligned}
P C= & \left\{x: \Omega \times[0, T] \longrightarrow X, x \in C\left(J_{k}, L^{2}(\Omega, X)\right), k=1, \ldots, m\right. \text { such that } \\
& x\left(t_{k}^{+}, .\right) \text {and } x\left(t_{k}^{-}, .\right) \text {exist with } x\left(t_{k}^{-}, .\right)=x\left(t_{k}, .\right) \text { and } \\
& \left.\sup _{t \in[0, T]} E\|x(t, .)\|^{2}<\infty \text { almost surely }\right\}
\end{aligned}
$$

endowed with the norm

$$
\|x\|_{P C}=\sup _{s \in[0, T]}\left(E\|x(s, .)\|^{2}\right)^{\frac{1}{2}} .
$$

It is not difficult to check that $P C$ is a Banach space with norm $\|\cdot\|_{P C}$.

Consider the Banach space

$$
P C_{b}=\left\{y \in P C_{*}\left(\mathbb{R}^{+} \times \Omega, X\right): y \text { is bounded almost surely }\right\},
$$

where

$P C_{*}\left(\mathbb{R}^{+} \times \Omega, X\right)=\left\{y:[0, \infty) \times \Omega \rightarrow X, y_{k} \in C\left(\left(t_{k}, t_{k+1}\right), L^{2}(\Omega, X)\right), k=0,1,2 \ldots\right.$, $y\left(t_{k}^{-}\right)$and $y\left(t_{k}^{+}\right)$exist and satisfy $y\left(t_{k}\right)=y\left(t_{k}^{-}\right)$for $\left.k=1, \ldots\right\}$

and $y_{k}:=y_{\mid\left(t_{k}, t_{k+1}\right]}$. Endowed with the norm

$$
\|y\|_{b}^{2}=\sup \left\{E|y(t)|_{X}^{2}: t \in[0, \infty)\right\},
$$

$P C_{b}$ is a Banach space.

For the study of this problem we first introduce the following hypotheses:

$\left(H_{7}\right)$ There exist $M>0$ and $\gamma>0$ such that

$$
\|S(t)\| \leq M e^{-\gamma t}
$$

for all $t>0$.

$\left(H_{8}\right)$ There exist nonnegative numbers $a_{i}$ and $b_{i}$ for each $i \in\{1,2\}$, and for all $0 \leq$ $\delta \leq \gamma$ it holds

$$
\begin{aligned}
\int_{0}^{t} e^{\delta s} E \mid f^{i}(s, x(s), y(s)) & -\left.f^{i}(s, \bar{x}(s), \bar{y}(s))\right|_{X} ^{2} d s \leq a_{i} \int_{0}^{t} e^{\delta s} E|x(s)-\bar{x}(s)|_{X}^{2} d s \\
& +b_{i} \int_{0}^{t} e^{\delta s} E|y(s)-\bar{y}(s)|_{X}^{2}
\end{aligned}
$$

and

$$
\int_{0}^{\infty} e^{\gamma s}\left|f^{i}(s, 0,0)\right|_{X}^{2} d s<\infty
$$

for all $x, y, \bar{x}, \bar{y} \in P C_{b}$. 
$\left(H_{9}\right)$ There exist functions $\alpha_{i}, \beta_{i} \in C\left(\mathbb{R}^{+}, \mathbb{R}^{+}\right), i=1,2$ such that, for all $0 \leq \delta \leq \gamma$, $x, y, \bar{x}, \bar{y} \in P C_{b}$ and $t \in \mathbb{R}^{+}$, we have

$$
\begin{aligned}
\int_{0}^{t} e^{\delta s} E\left\|\sigma^{i}(s, x(s), y(s))-\sigma^{i}(s, \bar{x}(s), \bar{y}(s))\right\|^{2} d s \leq & \alpha_{i}(t) \int_{0}^{t} e^{\delta s} E|x(s)-\bar{x}(s)|_{X}^{2} d s \\
& +\beta_{i}(t) \int_{0}^{t} e^{\delta s} E|y(s)-\bar{y}(s)|_{X}^{2} d s
\end{aligned}
$$

and

$$
\int_{0}^{\infty} e^{\gamma s} E\left\|\sigma^{i}(s, 0,0)\right\|^{2} d s<\infty, \sup _{t \in \mathbb{R}^{+}} t^{2 H-1} \alpha_{i}(t)<\infty \sup _{t \in \mathbb{R}^{+}} t^{2 H-1} \beta_{i}(t)<\infty .
$$

$\left(H_{10}\right)$ there exist constants $d_{k} \geq 0$ and $\bar{d}_{k} \geq 0, k=1, \ldots$, such that

$$
\begin{aligned}
& E\left|I_{k}(x)-I_{k}(\bar{x})\right|_{X}^{2} \leq d_{k} E|x-\bar{x}|_{X}^{2} \\
& E\left|\bar{I}_{k}(y)-\bar{I}_{k}(\bar{y})\right|_{X}^{2} \leq \bar{d}_{k} E|y-\bar{y}|_{X}^{2},
\end{aligned}
$$

and

$$
\sum_{k=1}^{\infty} e^{\gamma t_{k}} E\left|I_{k}(0)\right|_{X}^{2}<\infty, 4 M^{2} \sum_{k=1}^{\infty} d_{k}<1
$$

and

$$
\sum_{k=1}^{\infty} e^{\gamma t_{k}} E\left|\bar{I}_{k}(0)\right|_{X}^{2}<\infty, 4 M^{2} \sum_{k=1}^{\infty} \bar{d}_{k}<1
$$

for all $x, y, \bar{x}, \bar{y} \in P C_{b}$.

Theorem 6.1. Assume $\left(H_{7}\right)-\left(H_{10}\right)$ hold and that $\gamma>A_{2}>0$ where

$$
\begin{aligned}
A_{2} & =\max \left\{\frac{4 M^{2} c_{2}(H) H(2 H-1)\left(\bar{c}_{2}+\bar{c}_{1}\right)}{\widetilde{k}}, \frac{4 \gamma^{-1} M^{2}\left(c_{2}+c_{1}\right)}{\widetilde{k}}\right\}, \\
c_{i} & =\max \left\{a_{i}, b_{i}\right\}, \bar{c}_{i}=\max \left\{\sup _{t \in \mathbb{R}_{+}} t^{2 H-1} \alpha_{i}(t), \sup _{t \in \mathbb{R}_{+}} t^{2 H-1} \beta_{i}(t)\right\},
\end{aligned}
$$

and

$$
\bar{k}_{*}=4 M^{2} \sum_{k=1}^{\infty} \bar{d}_{k}, k_{*}=4 M^{2} \sum_{k=1}^{\infty} d_{k}, \frac{1}{\widetilde{k}}=\max \left(\frac{1}{1-k_{*}}, \frac{1}{1-\bar{k}_{*}}\right) .
$$

Then, there exists a unique mild solution $(x, y)$ to problem (1.1) which converges to zero in mean square, i.e.

$$
\lim _{t \rightarrow+\infty}\left(\begin{array}{l}
E\|x(t)\|^{2} \\
E\|y(t)\|^{2}
\end{array}\right)=\left(\begin{array}{l}
0 \\
0
\end{array}\right)
$$


Proof. It is clear that each mild solution to Problem (1.1) is a fixed point of the operator $N$ defined in Theorem 4.1. By using $\left(H_{7}\right)-\left(H_{10}\right)$, we can easily prove that $N\left(P C_{b}\right) \subset P C_{b}$, and from Perov's fixed there exists a unique $(x, y) \in P C_{b} \times P C_{b}$ which is a fixed point of $N$. Now, we show that

$$
\begin{aligned}
\lim _{t \rightarrow+\infty} E|N(x, y)|_{X}^{2} & =\lim _{t \rightarrow+\infty}\left(E\left|N_{1}(x, y)\right|_{X}^{2}, E\left|N_{2}(x, y)\right|_{X}^{2}\right) \\
& =\lim _{t \rightarrow+\infty}\left(E|x(t)|_{X}^{2}, E|y(t)|_{X}^{2}\right) \\
& =(0,0) .
\end{aligned}
$$

First, observe that we have

$$
\begin{aligned}
E|x(t)|_{X}^{2} \leq & 4 E\left|S(t) x_{0}\right|_{X}^{2}+4 E\left|\int_{0}^{t} S(t-s) f^{1}(s, x(s), y(s)) d s\right|_{X}^{2} \\
& +4 E\left|\sum_{l=1}^{\infty} \int_{0}^{t} S(t-s) \sigma_{l}^{1}(s, x(s), y(s)) d B_{l}^{H}(s)\right|_{X}^{2} \\
& +4 E\left|\sum_{0<t_{k}<t} S\left(t-t_{k}\right) I_{k}\left(x\left(t_{k}\right)\right)\right|_{X}^{2}
\end{aligned}
$$

and

$$
\begin{aligned}
E|y(t)|_{X}^{2} \leq & 4 E\left|S(t) y_{0}\right|_{X}^{2}+4 E\left|\int_{0}^{t} S(t-s) f^{1}(s, x(s), y(s)) d s\right|_{X}^{2} \\
& +4 E\left|\sum_{l=1}^{\infty} \int_{0}^{t} S(t-s) \sigma_{l}^{1}(s, x(s), y(s)) d B_{l}^{H}(s)\right|_{X}^{2} \\
& +4 E\left|\sum_{0<t_{k}<t} S\left(t-t_{k}\right) \bar{I}_{k}\left(y\left(t_{k}\right)\right)\right|_{X}^{2} .
\end{aligned}
$$

Therefore, by Lemma 2.8 and $\left(H_{7}\right)$,

$$
\begin{aligned}
E|x(t)|_{X}^{2} \leq & 4 M^{2} e^{-2 \gamma t} E\left|x_{0}\right|_{X}^{2}+4 \gamma^{-1} M^{2} \int_{0}^{t} e^{-\gamma(t-s)} E\left|f^{1}(s, x(s), y(s))\right|_{X}^{2} d s \\
& +4 M^{2} c_{2}(H) H(2 H-1) t^{2 H-1} \sum_{l=1}^{\infty} \int_{0}^{t} e^{-2 \gamma(t-s)}\left\|\sigma_{l}^{1}(s, x(s), y(s))\right\|^{2} d s \\
& +4 M^{2} \sum_{0<t_{k}<t} e^{-2 \gamma\left(t-t_{k}\right)} E\left|I_{k}\left(x\left(t_{k}\right)\right)\right|_{X}^{2}
\end{aligned}
$$


and, consequently,

$$
\begin{aligned}
e^{\gamma t} E|x(t)|_{X}^{2} \leq & 4 M^{2} E\left|x_{0}\right|_{X}^{2}+4 \gamma^{-1} M^{2} \int_{0}^{t} e^{\gamma s} E\left|f^{1}(s, x(s), y(s))\right|_{X}^{2} d s \\
& +4 M^{2} c_{2}(H) H(2 H-1) t^{2 H-1} \sum_{l=1}^{\infty} \int_{0}^{t} e^{\gamma s}\left\|\sigma_{l}^{1}(s, x(s), y(s))\right\|^{2} d s \\
& +4 M^{2} \sum_{0<t_{k}<t} e^{\gamma t_{k}} E\left|I_{k}\left(x\left(t_{k}\right)\right)\right|_{X}^{2} .
\end{aligned}
$$

Thanks to the fact that $A_{2}-\gamma<0$ we can choose $\theta>0$ such that $\eta=A_{2}-\gamma+\theta<0$, and for these constants we have

$$
\begin{aligned}
e^{(\gamma-\theta) t} E|x(t)|_{X}^{2} \leq & 4 M^{2} e^{-\theta t} E\left|x_{0}\right|_{X}^{2}+4 e^{-\theta t} \gamma^{-1} M^{2} \int_{0}^{t} e^{\gamma s} E\left|f^{1}(s, x(s), y(s))\right|_{X}^{2} d s \\
& +4 M^{2} c_{2}(H) H(2 H-1) t^{2 H-1} e^{-\theta t} \sum_{l=1}^{\infty} \int_{0}^{t} e^{\gamma s}\left\|\sigma_{l}^{1}(s, x(s), y(s))\right\|^{2} d s \\
& +4 M^{2} e^{-\theta t} \sum_{0<t_{k}<t} e^{\gamma t_{k}} E\left|I_{k}\left(x\left(t_{k}\right)\right)\right|_{X}^{2} \\
\leq & 4 M^{2} e^{-\theta t} E\left|x_{0}\right|_{X}^{2}+4 \gamma^{-1} M^{2} \int_{0}^{t} e^{(\gamma-\theta) s} E\left|f^{1}(s, x(s), y(s))\right|_{X}^{2} d s \\
& +4 M^{2} c_{H} H(2 H-1) t^{2 H-1} \int_{0}^{t} e^{(\gamma-\theta) s} E\left\|\sigma^{1}(s, x(s), y(s))\right\|^{2} d s \\
& +4 M^{2} e^{-\theta t} \sum_{0<t_{k}<t} e^{\gamma t_{k}} E\left|I_{k}\left(x\left(t_{k}\right)\right)\right|_{X}^{2} \\
= & 4 \sum_{i=1}^{4} P_{i} .
\end{aligned}
$$

Similarly,

$$
\begin{aligned}
e^{(\gamma-\theta) t} E|y(t)|_{X}^{2} \leq & 4 M^{2} e^{-\theta t} E\left|y_{0}\right|_{X}^{2}+4 \gamma^{-1} M^{2} \int_{0}^{t} e^{(\gamma-\theta) s} E\left|f^{2}(s, x(s), y(s))\right|_{X}^{2} d s \\
& +4 M^{2} c_{2}(H) H(2 H-1) t^{2 H-1} \int_{0}^{t} e^{(\gamma-\theta) s} E\left\|\sigma^{2}(s, x(s), y(s))\right\|^{2} d s \\
& +4 M^{2} e^{-\theta t} \sum_{0<t_{k}<t} e^{\gamma t_{k}} E\left|\bar{I}_{k}\left(y\left(t_{k}\right)\right)\right|_{X}^{2} \\
= & 4 \sum_{i=1}^{4} \bar{P}_{i} .
\end{aligned}
$$


Now it is easy to see, for any $t \geq 0$, that

$$
\begin{aligned}
P_{2} & =\int_{0}^{t} e^{(\gamma-\theta) s} E\left|f^{1}(s, x(s), y(s))\right|_{X}^{2} d s \\
& \leq \int_{0}^{t} e^{(\gamma-\theta) s} E\left|f^{1}(s, x(s), y(s))-f^{1}(s, 0,0)+f^{1}(s, 0,0)\right|_{X}^{2} d s \\
& \leq \int_{0}^{t} e^{(\gamma-\theta) s} E\left|f^{1}(s, x(s), y(s))-f^{1}(s, 0,0)\right|_{X}^{2} d s+\int_{0}^{t} e^{(\gamma-\theta) s} E\left|f^{1}(s, 0,0)\right|_{X}^{2} d s \\
& \leq a_{1} \int_{0}^{t} e^{(\gamma-\theta) s} E|x(s)|_{X}^{2} d s+b_{1} \int_{0}^{t} e^{(\gamma-\theta) s} E|y(s)|_{X}^{2} d s+\int_{0}^{t} e^{(\gamma-\theta) s} E\left|f^{1}(s, 0,0)\right|_{X}^{2} d s,
\end{aligned}
$$

and similarly,

$$
\begin{aligned}
\bar{P}_{2} & =\int_{0}^{t} e^{(\gamma-\theta) s} E\left|f^{2}(s, x(s), y(s))\right|_{X}^{2} d s \\
& \leq a_{2} \int_{0}^{t} e^{(\gamma-\theta) s} E|x(s)|_{X}^{2} d s+b_{2} \int_{0}^{t} e^{(\gamma-\theta) s} E|y(s)|_{X}^{2} d s+\int_{0}^{t} e^{(\gamma-\theta) s} E\left|f^{2}(s, 0,0)\right|_{X}^{2} d s .
\end{aligned}
$$

By condition $\left(H_{8}\right)$ we deduce the existence of positive constants $K_{1}, \bar{K}_{1}$ such that

$$
4 \gamma^{-1} M^{2} \int_{0}^{t} e^{(\gamma-\theta) s} E\left|f^{1}(s, 0,0)\right|_{X}^{2} d s \leq K_{1}
$$

and

$$
4 \gamma^{-1} M^{2} \int_{0}^{t} e^{(\gamma-\theta) s} E\left|f^{2}(s, 0,0)\right|_{X}^{2} d s \leq \bar{K}_{1}
$$

As for the term $P_{3}$ we have

$$
\begin{aligned}
P_{3} & =\int_{0}^{t} e^{(\gamma-\theta) s} E\left\|\sigma^{1}(s, x(s), y(s))\right\|^{2} d s \\
& \leq \int_{0}^{t} e^{(\gamma-\theta) s} E\left\|\sigma^{1}(s, x(s), y(s))-\sigma^{1}(s, 0,0)+\sigma^{1}(s, 0,0)\right\|^{2} d s \\
& \leq \int_{0}^{t} e^{(\gamma-\theta) s} E\left\|\sigma^{1}(s, x(s), y(s))-\sigma^{1}(s, 0,0)\right\|^{2} d s+\int_{0}^{t} e^{(\gamma-\theta) s} E\left\|\sigma^{1}(s, 0,0)\right\|^{2} d s \\
& \leq \alpha_{1}(t) \int_{0}^{t} e^{(\gamma-\theta) s} E|x(s)|_{X}^{2} d s+\beta_{1}(t) \int_{0}^{t} e^{(\gamma-\theta) s} E|y(s)|_{X}^{2} d s+\int_{0}^{t} e^{(\gamma-\theta) s} E\left\|\sigma^{1}(s, 0,0)\right\|^{2} d s,
\end{aligned}
$$

and similarly,

$$
\begin{aligned}
\bar{P}_{3} & =\int_{0}^{t} e^{(\gamma-\theta) s} E\left\|\sigma^{2}(s, x(s), y(s))\right\|^{2} d s \\
& \leq \alpha_{2}(t) \int_{0}^{t} e^{(\gamma-\theta) s} E|x(s)|_{X}^{2} d s+\beta_{2}(t) \int_{0}^{t} e^{(\gamma-\theta) s} E|y(s)|_{X}^{2} d s+\int_{0}^{t} e^{(\gamma-\theta) s} E\left\|\sigma^{1}(s, 0,0)\right\|^{2} d s .
\end{aligned}
$$


From $\left(H_{9}\right)$ we deduce the existence of positive constants $K_{2}, \bar{K}_{2}$, such that

$$
4 M^{2} c_{2}(H) H(2 H-1) t^{2 H-1} \int_{0}^{t} e^{(\gamma-\theta) s} E\left\|\sigma^{1}(s, 0,0)\right\|^{2} d s \leq K_{2}
$$

and

$$
4 M^{2} c_{2}(H) H(2 H-1) t^{2 H-1} \int_{0}^{t} e^{(\gamma-\theta) s} E\left\|\sigma^{2}(s, 0,0)\right\|^{2} d s \leq \bar{K}_{2} .
$$

And for the last terms

$$
\begin{aligned}
P_{4} & =e^{-\theta t} \sum_{0<t_{k}<t} e^{\gamma t_{k}} E\left|I_{k}\left(x\left(t_{k}\right)\right)\right|_{X}^{2} \\
& \leq e^{-\theta t} \sum_{0<t_{k}<t} e^{\gamma t_{k}} E\left|I_{k}\left(x\left(t_{k}\right)\right)-I_{k}(0)+I_{k}(0)\right|_{X}^{2} \\
& \leq e^{-\theta t} \sum_{0<t_{k}<t} e^{\gamma t_{k}} E\left|I_{k}\left(x\left(t_{k}\right)\right)-I_{k}(0)\right|_{X}^{2}+e^{-\theta t} \sum_{0<t_{k}<t} e^{\gamma t_{k}} E\left|I_{k}(0)\right|_{X}^{2} \\
& \leq e^{-\theta t} \sum_{0<t_{k}<t} e^{\gamma t_{k}} d_{k} E\left|x\left(t_{k}\right)\right|_{X}^{2}+e^{-\theta t} \sum_{0<t_{k}<t} e^{\gamma t_{k}} E\left|I_{k}(0)\right|_{X}^{2} \\
& \leq e^{(\gamma-\theta) t} \sum_{0<t_{k}<t} e^{\gamma\left(t_{k}-t\right)} d_{k} E\left|x\left(t_{k}\right)\right|_{X}^{2}+e^{-\theta t} \sum_{0<t_{k}<t} e^{\gamma t_{k}} E\left|I_{k}(0)\right|_{X}^{2},
\end{aligned}
$$

and analogously

$$
\begin{aligned}
\bar{P}_{4} & =e^{-\theta t} \sum_{0<t_{k}<t} e^{\gamma t_{k}} E\left|\bar{I}_{k}\left(y\left(t_{k}\right)\right)\right|_{X}^{2} \\
& \leq e^{(\gamma-\theta) t} \sum_{0<t_{k}<t} e^{\gamma\left(t_{k}-t\right)} d_{k} E\left|x\left(t_{k}\right)\right|_{X}^{2}+e^{-\theta t} \sum_{0<t_{k}<t} e^{\gamma t_{k}} E\left|\bar{I}_{k}(0)\right|_{X}^{2} .
\end{aligned}
$$

Now, thanks to $\left(H_{10}\right)$ we have the existence of positive constants $K_{3}, \bar{K}_{3}$ such that, for all $t \geq 0$,

$$
e^{-\theta t} \sum_{0<t_{k}<t} e^{\gamma t_{k}} E\left|I_{k}(0)\right|_{X}^{2} \leq K_{3} \quad \text { and } \quad e^{-\theta t} \sum_{0<t_{k}<t} e^{\gamma t_{k}} E\left|\bar{I}_{k}(0)\right|_{X}^{2} \leq \bar{K}_{3},
$$

which further imply

$$
\begin{aligned}
e^{(\gamma-\theta) t} E|x(t)|_{X}^{2} \leq & 4 M^{2} e^{-\theta t} E\left|x_{0}\right|_{X}^{2}+\sum_{i=1}^{3} K_{i}+4 \gamma^{-1} M^{2} a_{1} \int_{0}^{t} e^{(\gamma-\theta) s} E|x(s)|_{X}^{2} d s \\
& +4 \gamma^{-1} M^{2} b_{1} \int_{0}^{t} e^{(\gamma-\theta) s} E|y(s)|_{X}^{2} d s \\
& +4 M^{2} c_{2}(H) H(2 H-1) t^{2 H-1} \alpha_{1} \int_{0}^{t} e^{(\gamma-\theta) s} E|x(s)|_{X}^{2} d s \\
& +4 M^{2} c_{2}(H) H(2 H-1) t^{2 H-1} \beta_{1} \int_{0}^{t} e^{(\gamma-\theta) s} E|y(s)|_{X}^{2} d s \\
& +4 M^{2} e^{(\gamma-\theta) t} \sum_{0<t_{k}<t} e^{\gamma\left(t_{k}-t\right)} d_{k} E\left|x\left(t_{k}\right)\right|_{X}^{2}
\end{aligned}
$$


and similarly

$$
\begin{aligned}
e^{(\gamma-\theta) t} E|y(t)|_{X}^{2} \leq & 4 M^{2} e^{-\theta t} E\left|y_{0}\right|_{X}^{2}+\sum_{i=1}^{3} \bar{K}_{i}+4 \gamma^{-1} M^{2} a_{2} \int_{0}^{t} e^{(\gamma-\theta) s} E|x(s)|_{X}^{2} d s \\
& +4 \gamma^{-1} M^{2} b_{2} \int_{0}^{t} e^{(\gamma-\theta) s} E|y(s)|_{X}^{2} d s \\
& +4 M^{2} c_{2}(H) H(2 H-1) t^{2 H-1} \alpha_{2} \int_{0}^{t} e^{(\gamma-\theta) s} E|x(s)|_{X}^{2} d s \\
& +4 M^{2} c_{2}(H) H(2 H-1) t^{2 H-1} \beta_{2} \int_{0}^{t} e^{(\gamma-\theta) s} E|y(s)|_{X}^{2} d s \\
& +4 M^{2} e^{(\gamma-\theta) t} \sum_{0<t_{k}<t} e^{\gamma\left(t_{k}-t\right)} \bar{d}_{k} E\left|y\left(t_{k}\right)\right|_{X}^{2} .
\end{aligned}
$$

Consider functions $\mu, \bar{\mu}$ defined on $[0,+\infty)$ by

$$
\mu(t)=\sup \left\{E|x(s)|_{X}^{2}: 0 \leq s \leq t\right\}, \quad \bar{\mu}(t)=\sup \left\{E|y(s)|_{X}^{2}: 0 \leq s \leq t\right\} .
$$

Together with $\left(H_{9}\right)$, it follows that, for each $t>0$, the constant $k_{*}(t)=4 M^{2} \sum_{0<t_{k}<t} e^{\gamma\left(t_{k}-t\right)} d_{k} \leq$ $k_{*}<1$. Then

$$
\begin{aligned}
e^{(\gamma-\theta) t} \mu(t) & \leq \frac{4 M^{2}}{1-k_{*}} e^{-\theta t} E\left|x_{0}\right|_{X}^{2}+\sum_{i=1}^{3} \frac{K_{i}}{1-k_{*}}+\frac{4 \gamma^{-1} M^{2} a_{1}}{1-k_{*}} \int_{0}^{t} e^{(\gamma-\theta) s} \mu(s) d s \\
& +4 \frac{\gamma^{-1} M^{2} b_{1}}{1-k_{*}} \int_{0}^{t} e^{(\gamma-\theta) s} \bar{\mu}(s) d s \\
& +\frac{4 M^{2} c_{2}(H) H(2 H-1) t^{2 H-1} \alpha_{1}(t)}{1-k_{*}} \int_{0}^{t} e^{(\gamma-\theta) s} \mu(s) d s \\
& +\frac{4 M^{2} c_{2}(H) H(2 H-1) t^{2 H-1} \beta_{1}(t)}{1-k_{*}} \int_{0}^{t} e^{(\gamma-\theta) s} \bar{\mu}(s) d s
\end{aligned}
$$

Analogously, the constant $\bar{k}_{*}(t)=4 M^{2} \sum_{0<t_{k}<t} e^{\gamma\left(t_{k}-t\right)} \bar{d}_{k} \leq \bar{k}_{*}<1$ and we have

$$
\begin{aligned}
e^{(\gamma-\theta) t} \bar{\mu}(t) \leq & \frac{4 M^{2}}{1-\bar{k}_{*}} e^{-\theta t} E\left|y_{0}\right|_{X}^{2}+\sum_{i=1}^{3} \frac{\bar{K}_{i}}{1-\bar{k}_{*}}+\frac{4 \gamma^{-1} M^{2} a_{2}}{1-\bar{k}_{*}} \int_{0}^{t} e^{(\gamma-\theta) s} \mu(s) d s \\
+ & 4 \frac{\gamma^{-1} M^{2} b_{2}}{1-\bar{k}_{*}} \int_{0}^{t} e^{(\gamma-\theta) s} \bar{\mu}(s) d s \\
& +\frac{4 M^{2} c_{2}(H) H(2 H-1) t^{2 H-1} \alpha_{2}}{1-\bar{k}_{*}} \int_{0}^{t} e^{(\gamma-\theta) s} \mu(s) d s \\
+ & \frac{4 M^{2} c_{2}(H) H(2 H-1) t^{2 H-1} \beta_{2}}{1-\bar{k}_{*}} \int_{0}^{t} e^{(\gamma-\theta) s} \bar{\mu}(s) d s
\end{aligned}
$$


Therefore,

$$
\begin{aligned}
e^{(\gamma-\theta) t}(\mu(t)+\bar{\mu}(t)) & \leq \frac{4 M^{2}}{1-k_{*}} e^{-\theta t} E\left|x_{0}\right|_{X}^{2}+\frac{4 M^{2}}{1-\bar{k}_{*}} e^{-\theta t} E\left|y_{0}\right|_{X}^{2}+\sum_{i=1}^{3} \frac{K_{i}}{1-k_{*}}+\sum_{i=1}^{3} \frac{\bar{K}_{i}}{1-\bar{k}_{*}} \\
& +\frac{4 \gamma^{-1} M^{2}}{1-k_{*}}\left(a_{1} \int_{0}^{t} e^{(\gamma-\theta) s} \mu(s) d s+b_{1} \int_{0}^{t} e^{(\gamma-\theta) s} \bar{\mu}(s) d s\right) \\
& +\frac{4 M^{2} c_{2}(H) H(2 H-1)}{1-k_{*}}\left(t^{2 H-1} \alpha_{1}(t) \int_{0}^{t} e^{(\gamma-\theta) s} \mu(s) d s\right. \\
& \left.+t^{2 H-1} \beta_{1}(t) \int_{0}^{t} e^{(\gamma-\theta) s} \bar{\mu}(s) d s\right) \\
& +\frac{4 \gamma^{-1} M^{2}}{1-\bar{k}_{*}}\left(a_{2} \int_{0}^{t} e^{(\gamma-\theta) s} \mu(s) d s+b_{2} \int_{0}^{t} e^{(\gamma-\theta) s} \bar{\mu}(s) d s\right) \\
& +\frac{4 M^{2} c_{2}(H) H(2 H-1)}{1-\bar{k}_{*}}\left(t^{2 H-1} \alpha_{2}(t) \int_{0}^{t} e^{(\gamma-\theta) s} \mu(s) d s\right. \\
& \left.+t^{2 H-1} \beta_{2}(t) \int_{0}^{t} e^{(\gamma-\theta) s} \bar{\mu}(s) d s\right),
\end{aligned}
$$

the maximum being taken componentwise for each $i=1,2$, and

$$
\begin{aligned}
c_{i}=\max \left\{a_{i}, b_{i}\right\}, \bar{c}_{i}= & \max \left\{t^{2 H-1} \alpha_{i}(t), t^{2 H-1} \beta_{i}(t)\right\}, \quad \frac{1}{\widetilde{k}}=\max \left\{\frac{1}{1-\bar{k}_{*}}, \frac{1}{1-k_{*}}\right\} \\
e^{(\gamma-\theta) t}(\mu(t)+\bar{\mu}(t)) \leq & \frac{4 M^{2}}{\widetilde{k}} e^{-\theta t}\left(E\left|x_{0}\right|_{X}^{2}+E\left|y_{0}\right|_{X}^{2}\right)+\sum_{i=1}^{3} \frac{K_{i}+\bar{K}_{i}}{\widetilde{k}} \\
& +\frac{4 \gamma^{-1} M^{2} c_{1}}{\widetilde{k}} \int_{0}^{t} e^{(\gamma-\theta) s}(\mu(s)+\bar{\mu}(s)) d s \\
& +\frac{4 M^{2} c_{2}(H) H(2 H-1) \bar{c}_{1}}{\widetilde{k}} \int_{0}^{t} e^{(\gamma-\theta) s}(\mu(s)+\bar{\mu}(s)) d s \\
& +\frac{4 \gamma^{-1} M^{2} c_{2}}{\widetilde{k}} \int_{0}^{t} e^{(\gamma-\theta) s}(\mu(s)+\bar{\mu}(s)) d s \\
& +\frac{4 M^{2} c_{2}(H) H(2 H-1) \bar{c}_{2}}{\widetilde{k}} \int_{0}^{t} e^{(\gamma-\theta) s}(\mu(s)+\bar{\mu}(s)) d s
\end{aligned}
$$

where

$$
A_{2}=\max \left\{\frac{4 M^{2} c_{2}(H) H(2 H-1)\left(\bar{c}_{2}+\bar{c}_{1}\right)}{\widetilde{k}}, \frac{4 \gamma^{-1} M^{2}\left(c_{2}+c_{1}\right)}{\widetilde{k}}\right\}
$$

and

$$
A_{1}=\frac{4 M^{2}}{\widetilde{k}} e^{-\theta t}\left(E\left|x_{0}\right|_{X}^{2}+E\left|y_{0}\right|_{X}^{2}\right)+\sum_{i=1}^{3} \frac{K_{i}+\bar{K}_{i}}{\widetilde{k}}
$$




$$
e^{(\gamma-\theta) t}(\mu(t)+\bar{\mu}(t)) \leq A_{1}+A_{2} \int_{0}^{t} e^{(\gamma-\theta) s}(\mu(s)+\bar{\mu}(s)) d s,
$$

where $A_{1}$ is a suitable positive constant. Gronwall's Lemma conduces us to

$$
e^{(\gamma-\theta) t}(\mu(t)+\bar{\mu}(t)) \leq A_{1} e^{A_{2} t}
$$

and, consequently,

$$
\mu(t)+\bar{\mu}(t) \leq A_{1} e^{\left(A_{2}-\gamma+\theta\right) t}=A_{1} e^{\eta t} .
$$

Then

$$
\lim _{t \rightarrow \infty}[\mu(t)+\bar{\mu}(t)]=0 \Rightarrow \lim _{t \rightarrow \infty} E|x(t)|_{X}^{2}=0, \lim _{t \rightarrow \infty} E|y(t)|_{X}^{2}=0 .
$$

The proof is therefore complete.

\section{An example}

In this section we present an example to illustrate the usefulness and applicability of our results. We consider with finite or infinite fractional Browian motion.

Example 7.1. Consider the following couple stochastic partial differential equation with impulsive effects

$$
\left\{\begin{aligned}
d u(t, \xi)= & \frac{\partial^{2}}{\partial \xi^{2}} u(t, \xi)+F(t, u(t, \xi), v(t, \xi)) \\
& +\sigma(t) \frac{d B_{Q}^{H}}{d t}, \quad t \geq 0, \quad t \neq t_{k}, \quad 0 \leq \xi \leq \pi, \\
d v(t, \xi)= & \frac{\partial^{2}}{\partial \xi^{2}} v(t, \xi)+G(t, u(t, \xi), v(t, \xi)) \\
& +\sigma(t) \frac{d B_{Q}^{H}}{d t}, \quad t \geq 0, \quad t \neq t_{k}, \quad 0 \leq \xi \leq \pi, \quad k=1, \cdots, m, \\
u\left(t_{k}^{+}, \xi\right)= & u\left(t_{k}^{-}, \xi\right)=\alpha_{k} u\left(t_{k}^{-}, \xi\right), \quad k=1, \cdots, m, \\
v\left(t_{k}^{+}, \xi\right)= & v\left(t_{k}^{-}, \xi\right)=\bar{\alpha}_{k} v\left(t_{k}^{-}, \xi\right), \quad k=1, \cdots, \\
u(t, 0)= & u(t, \pi)=0, t \geq 0, \\
v(t, 0)= & v(t, \pi)=0, t \geq 0, \\
u(0, \xi)= & u_{0}(\xi), 0 \leq \xi \leq \pi, \\
v(0, \xi)= & v_{0}(\xi), \quad 0 \leq \xi \leq \pi,
\end{aligned}\right.
$$

where $\alpha_{k}>0, B_{Q}^{H}$ denotes a fractional Brownian motion, $G, F:[0, \pi] \times \mathbb{R} \times \mathbb{R} \rightarrow \mathbb{R}$ are continuous functions.

$$
\begin{aligned}
& \text { Let } \\
& \qquad \begin{aligned}
x(t)(\xi)=u(t, \xi), y(t)(\xi)=v(t, \xi) \quad t \in J, \quad \xi \in[0, \pi], \\
I_{k}\left(x\left(t_{k}\right)\right)(\xi)=\alpha_{k} u\left(t_{k}^{-}, \xi\right), \bar{I}_{k}\left(y\left(t_{k}\right)\right)(\xi)=\alpha_{k} v\left(t_{k}^{-}, \xi\right) \quad \xi \in[0, \pi], \quad k=1, \cdots, m, \\
f(t, x(t), y(t))(\xi)=F(t, u(t, \xi), v(t, \xi)), \quad, \quad \xi \in[0, \pi], \\
g(t, x(t), y(t))(\xi)=G(t, u(t, \xi), v(t, \xi)), \quad, \quad \xi \in[0, \pi] . \\
u_{0}(\xi)=u(0, \xi), v_{0}(\xi)=v(0, \xi) \quad, \quad \xi \in[0, \pi]
\end{aligned}
\end{aligned}
$$


Take $\mathcal{K}=\mathcal{H}=L^{2}([0, \pi])$. We define the operator $A$ by $A u=u^{\prime \prime}$, with domain $D(A)=\left\{u \in \mathcal{H}, u^{\prime}, u^{\prime \prime} \in \mathcal{H} \quad\right.$ and $\left.\quad u(0)=u(\pi)=0\right\}$.

Then, it is well known that

$$
A z=-\sum_{n=1}^{\infty} e^{-n^{2} t}\left\langle z, e_{n}\right\rangle e_{n}, \quad z \in \mathcal{H}
$$

and $A$ is the infinitesimal generator of an analytic semigroup $\{S(t)\}_{t \geq 0}$ on $\mathcal{H}$, which is given by

$$
S(t) u=\sum_{n=1}^{\infty} e^{-n^{2} t}\left\langle u, e_{n}\right\rangle e_{n}, u \in \mathcal{H}, \text { and } e_{n}(u)=(2 / \pi)^{1 / 2} \sin (n u), n=1,2, \cdots, \text { is }
$$

the orthogonal set of eigenvectors of $A$. The analytic semigroup $\{S(t)\}_{t>0}, t \in J$, is compact, and there exists a constant $M \geq 1$ such that $\|S(t)\|^{2} \leq M$.

In order to define the operator $Q: \mathcal{K} \longrightarrow \mathcal{K}$, we choose a sequence $\left\{\sigma_{n}\right\}_{n \geq 1} \subset \mathbb{R}^{+}$, set $Q e_{n}=\sigma_{n} e_{n}$, and assume that

$$
\operatorname{tr}(Q)=\sum_{n=1}^{\infty} \sqrt{\sigma_{n}}<\infty
$$

Define the process $B_{Q}^{H}(s)$ by

$$
B_{Q}^{H}=\sum_{n=1}^{\infty} \sqrt{\sigma_{n}} \gamma_{n}^{H}(t) e_{n}
$$

where $H \in(1 / 2,1)$, and $\left\{\gamma_{n}^{H}\right\}_{n \in \mathbb{N}}$ is a sequence of two-sided one-dimensional mutually independent fractional Brownian motions. Assume now that

(i) There exist some positive number $d_{k}, \bar{d}_{k} \quad k \in\{1, \cdots, m\}$ such that

$$
\left|I_{k}(\xi)\right| \leq d_{k}, \quad\left|\bar{I}_{k}(\xi)\right| \leq \bar{d}_{k}
$$

for any $\xi \in \mathbb{R}$.

(ii) The functions $f, g:[0, T] \times \mathcal{H} \longrightarrow \mathcal{H}$ defined by $f(t, u)()=.F(t, u()),. g(t, u)()=$. $G(t, u()$.$) are continuous and we impose suitable conditions on F$ and $G$ to verify assumption (H2) .

(iii) Assume that there exists an integrable function $\eta:[0, T] \longrightarrow \mathbb{R}^{+}$such that

$$
|F(t, x, y)|^{2} \leq \eta(t) \psi\left(|x|^{2}+|y|^{2}\right), \quad|G(t, x, y)|^{2} \leq \eta(t) \psi\left(|x|^{2}+|y|^{2}\right)
$$

for any $t \in[0, T]$ and $x, y \in \mathbb{R}$, where $\psi:[0, \infty) \longrightarrow(0, \infty)$ is continuous, nondecreasing and concave with

$$
\int_{1}^{\infty} \frac{d s}{\psi(s)}=+\infty
$$


(iv) The function $\sigma:[0, T] \longrightarrow L_{Q}^{2}(\mathcal{K}, \mathcal{H})$ is bounded, that is, there exists a positive constant $L$ such that

$$
\int_{0}^{T}\|\sigma(s)\|_{L_{Q}^{2}}^{2} d s<L, \quad \forall T>0
$$

Thus, problem (7.1) can be written in the abstract form

$$
\left\{\begin{array}{l}
d x(t)=[A x(t)+f(t, x, y)] d t+\sigma(t) d B_{Q}^{H}(t), \quad t \in J:=[0, T] \\
d y(t)=[A y(t)+g(t, x, y)] d t+\sigma(t) d B_{Q}^{H}(t), \quad t \in J:=[0, T] \\
x\left(t_{k}^{+}\right)-x\left(t_{k}\right)=I_{k}\left(x\left(t_{k}\right)\right), k=1, \ldots, m \\
y\left(t_{k}^{+}\right)-y\left(t_{k}\right)=I_{k}\left(y\left(t_{k}\right)\right), k=1, \ldots, m \\
x(0)=x_{0} \\
y(0)=y_{0} .
\end{array}\right.
$$

Thanks to these assumptions, it is straightforward to check that $\left(H_{1}\right)-\left(H_{6}\right)$ hold true and, then, assumptions in Theorem 4.2 are fulfilled, and we can conclude that system (7.1) possesses a mild solution on $[0, T]$.

In the case that we consider the problem for $t \in[0, \infty)$, we observe that

$\left(i^{\prime}\right)\|S(t)\| \leq e^{-\pi^{2} t}$,

$\left(i i^{\prime}\right)$ The functions $f, g:[0, \infty) \times \mathcal{H} \longrightarrow \mathcal{H}$ are continuous and under suitable conditions on $F$, assumption (H2) holds in $[0,+\infty)$.

$\left(\right.$ iii $\left.^{\prime}\right)$ The function $\sigma:[0,+\infty) \longrightarrow L_{Q}^{2}(\mathcal{K}, \mathcal{H})$ is bounded, that is, there exists a positive constant $L$ such that

$$
\int_{0}^{\infty} e^{\gamma s}\|\sigma(s)\|_{L_{Q}^{0}}^{2} d s<L .
$$

$\left(i v^{\prime}\right)$ There exist some positive number $d_{k}, \quad k \in\{1, \cdots, m, \cdots\}$ such that

$$
\left|I_{k}(\xi)\right| \leq d_{k}, \quad\left|\bar{I}_{k}(\xi)\right| \leq \bar{d}_{k} \text { and } \sum_{k=1}^{\infty} d_{k}<\infty, \sum_{k=1}^{\infty} \bar{d}_{k}<\infty
$$

for any $\xi \in \mathbb{R}$.

Thus the problem (7.1) can be written in the abstract form

$$
\left\{\begin{array}{l}
d x(t)=[A x(t)+f(t, x, y)] d t+\sigma(t) d B_{Q}^{H}(t), \quad t \in J:=[0, \infty) ; \\
d y(t)=[A y(t)+g(t, x, y)] d t+\sigma(t) d B_{Q}^{H}(t), \quad t \in J:=[0, \infty) ; \\
x\left(t_{k}^{+}\right)-x\left(t_{k}\right)=I_{k}\left(x\left(t_{k}\right)\right), k=1,2,3 \ldots \\
y\left(t_{k}^{+}\right)-y\left(t_{k}\right)=I_{k}\left(y\left(t_{k}\right)\right), \quad k=1,2,3 \ldots \\
x(0)=x_{0} \\
y(0)=y_{0} .
\end{array}\right.
$$


Corollary 7.1. Let $\sigma_{1}, \sigma_{2}:[0, \pi] \times L^{2}([0, \pi]) \times L^{2}([0, \pi]) \rightarrow L^{2}([0, \pi])$ be defined by

$$
\sigma^{1}(t, x, y)=\sum_{k=1}^{\infty} \sigma_{k}^{1}(t, x, y), \sigma^{2}(t, x, y)=\sum_{k=1}^{\infty} \sigma_{k}^{2}(t, x, y)
$$

where

$$
\sigma_{k}^{1}(t, x, y) v(s)=a_{2 k+1} \int_{0}^{t}\left[\sin k^{2} x(t)\right] f(s) d s+a_{2 k} \int_{0}^{t}\left[\cos k^{2} y(t)\right] f(s) d s, v \in L^{2}([0, \pi])
$$

and

$$
\sigma_{k}^{2}(t, x, y) v(s)=b_{2 k+1} \int_{0}^{t}\left[\sin k^{2} x(s)\right] f(s) d s+b_{2 k} \int_{0}^{t}\left[\cos k^{2} y(s)\right] f(s) d s, v \in L^{2}([0, \pi]) .
$$

If $\sum a_{k}^{2}<\infty, \sum b_{k}^{2}<\infty$, then for each $k \in \mathbb{N}$ we have

$$
\sigma_{k}^{1}, \sigma_{k}^{2} \in L_{Q}^{0}\left(L^{2}\left([0, \pi], L^{2}([0, \pi])\right)\right.
$$

and

$$
\sum_{l=1}^{\infty}\left\|\sigma_{l}^{i}(., x, y)\right\|_{L_{Q}^{0}}^{2}<\infty, i=1,2 .
$$

Proof. Clearly, for every $k \in \mathbb{N}, \sigma_{k}^{1}, \sigma_{k}^{2}$ are linear continuous operators. Let $\left(e_{i}\right)_{i \in \mathbb{N}}$ be an orthonormal basis of $L^{2}([0, \pi])$

$$
\begin{aligned}
& \left\|\sigma_{k}^{1}(t, x, y)\right\|_{L_{Q}}^{2} \\
& \quad=\sum_{k=1}^{\infty} \int_{0}^{\pi}\left(\left|\sqrt{\sigma_{n}} a_{2 k+1} \int_{0}^{t}\left[\sin k^{2} x(s)\right] e_{n}(s) d s+\sqrt{\sigma_{n}} a_{2 k} \int_{0}^{t}\left[\cos k^{2} y(s)\right] e_{n}(s) d s\right|^{2}\right) d t \\
& \quad \leq 2 \pi^{2}\left(a_{2 k+1}^{2}+a_{2 k}^{2}\right) \sum_{n=1}^{\infty} \sigma_{n} .
\end{aligned}
$$

Therefore

$$
\begin{gathered}
\left\|\sigma_{k}^{1}(t, x, y)\right\|_{L_{Q}^{0}} \leq \pi \sqrt{2\left(a_{2 k+1}^{2}+a_{2 k}^{2}\right)} \sum_{n=1}^{\infty} \sqrt{\sigma_{n}}<\infty . \\
\left\|\sigma_{k}^{1}(t, x, y)\right\|_{L_{Q}^{0}}^{2} \leq L_{*}\left(a_{2 k+1}^{2}+a_{2 k}^{2}\right), \quad L_{*}=2 \pi^{2} \sum_{n=1}^{\infty} \sqrt{\sigma_{n}} .
\end{gathered}
$$


We deduce

$$
\begin{aligned}
\left\|\sigma^{1}(t, x, y)\right\|^{2} & =\sum_{k=1}^{\infty}\left\|\sigma_{l}^{1}\right\|_{L_{Q}^{0}} \\
& \leq L_{*} \sum_{k=1}^{\infty}\left(a_{2 k+1}^{2}+a_{2 k}^{2}\right) \\
& \leq L_{*} \sum_{k=1}^{\infty} a_{k}^{2}<\infty .
\end{aligned}
$$

and

$$
\begin{aligned}
\left\|\sigma^{2}(t, x, y)\right\|^{2} & =\sum_{k=1}^{\infty}\left\|\sigma_{l}^{2}\right\|_{L_{Q}^{0}}^{2} \\
& \leq L_{*} \sum_{k=1}^{\infty} b_{k}^{2}<\infty .
\end{aligned}
$$

Hence

$$
E\left\|\sigma_{1}(t, x, y)\right\|^{2} \leq L_{*} \sum_{k=1}^{\infty} a_{k}^{2}+E\left(\|x\|_{L^{2}}^{2}+\|y\|_{L^{2}}^{2}\right) \text { for all } x, y \in L^{2}([0, \pi]),
$$

and

$$
E\left\|\sigma_{2}(t, x, y)\right\|^{2} \leq L_{*} \sum_{k=1}^{\infty} b_{k}^{2}+E\left(\|x\|_{L^{2}}^{2}+\|y\|_{L^{2}}^{2}\right) \text { for all } x, y \in L^{2}([0, \pi])
$$

Corollary 7.2. Let $K:[0, \pi] \times[0, \pi] \times \mathbb{R} \times \mathbb{R} \rightarrow \mathbb{R}$ be a continuous function such that there exist $a, b \geq 0$ such that

$$
|K(t, s, x, y)-K(t, s, \bar{x}, \bar{y})| \leq a|x-\bar{x}|+b|y-\bar{y}|, \quad \text { for all } x, \bar{x}, y, \bar{y} \in \mathbb{R} .
$$

Then $K_{*}:[0, \pi] \times L^{2}([0, \pi]) \times L^{2}([0, \pi]) \rightarrow L_{Q}^{0}\left(L^{2}([0, \pi]), L^{2}([0, \pi])\right)$ defined by

$$
K_{*}(t, x, y) f(t)=\int_{0}^{t} K(t, s, x(s), y(s)) f(s) d s, \quad f \in L^{2}([0, \pi])
$$

is a Hilbert-Schmidt operator and there exist $\alpha, \beta \geq 0$ such that $\left\|K_{*}(t,, x, y)-K(t, s, \bar{x}, \bar{y})\right\|_{L_{Q}^{0}} \leq \alpha\|x-\bar{x}\|_{L^{2}}+\beta\|y-\bar{y}\|_{L^{2}} \quad$ for each $x, \bar{x}, y, \bar{y} \in L^{2}([0, \pi])$. 
Proof. It is clear that $K_{*}(t, x, y)$ is a bounded linear operator. Let $\left(e_{i}\right)_{i \in \mathbb{N}}$ be an orthonormal basis of $L^{2}([0, \pi])$

$$
\begin{aligned}
& \left\|K_{*}(t, x, y)\right\|_{L_{Q}^{0}}^{2} \\
& \quad=\sum_{k=1}^{\infty} \int_{0}^{\pi}\left(\left|\sqrt{\sigma_{n}} \int_{0}^{t}[K(t, s, x(s), y(s))] e_{n}(s) d s\right|^{2}\right) d t \\
& \leq \sum_{n=1}^{\infty} \int_{0}^{\pi}\left(\sigma_{n}\left(\int_{0}^{\pi}[a|x(s)|+b|y(s)|]\left|e_{n}(s)\right| d s+\int_{0}^{\pi}|K(t, s, 0,0)|\left|e_{n}(s)\right| d s\right)^{2}\right) d t \\
& \leq 4 \pi a^{2} \sum_{n=1}^{\infty} \sigma_{n}\|x\|_{L^{2}}^{2}+4 \pi b^{2} \sum_{n=1}^{\infty} \sigma_{n}\|y\|_{L^{2}}^{2}+2 \pi^{2} \sup _{(t, s) \in[0, \pi] \times[0 ; \pi]}|K(t, s, 0,0)| \sum_{n=1}^{\infty} \sigma_{n} .
\end{aligned}
$$

Thus

$$
\left\|K_{*}(t, x, y)\right\|_{L_{Q}^{0}} \leq \alpha_{1}\|x\|_{L^{2}}+\beta_{1}\|y\|_{L^{2}}+\gamma_{1}<\infty
$$

where

$$
\alpha_{1}^{2}=4 \pi a^{2} \sum_{n=1}^{\infty} \sigma_{n}, \beta_{1}^{2}=4 \pi b^{2} \sum_{n=1}^{\infty} \sigma_{n}, \gamma_{1}^{2}=2 \pi^{2} \sup _{(t, s) \in[0, \pi] \times[0, \pi]}|K(t, s, 0,0)| \sum_{n=1}^{\infty} \sigma_{n} .
$$

We can easily prove $\left\|K_{*}(t,, x, y)-K(t, s, \bar{x}, \bar{y})\right\|_{L_{Q}}^{2} \leq \alpha\|x-\bar{x}\|_{L^{2}}^{2}+\beta\|y-\bar{y}\|_{L^{2}}^{2} \quad$ for each $x, \bar{x}, y, \bar{y} \in L^{2}([0, \pi])$, where $\alpha=\alpha_{1}^{2}, \beta=\beta_{1}^{2}$.

Corollary 7.3. Let $\left\{K_{n}\right\}_{n \in \mathbb{R}}:[0, \pi] \times[0, \pi] \times \mathbb{R} \times \mathbb{R} \rightarrow \mathbb{R}$ be a summability kernel on $[0, \pi]$ i.e it holds

$$
\sum_{n=1}^{\infty}\left\|K_{n}(t, ., x, y)\right\|_{L^{2}}^{2}<\infty
$$

and $K$ is a continuous function such that there exists $a_{n}, b_{n} \geq 0$ such that

$$
|K(t, s, x, y)-K(t, s, \bar{x}, \bar{y})| \leq a_{n}|x-\bar{x}|+b_{n}|y-\bar{y}|, \quad \text { for all } x, \bar{x}, y, \bar{y} \in \mathbb{R} .
$$

If

$$
\sum_{n=1}^{\infty}\left(a_{n}^{2}+b_{n}^{2}\right)<\infty
$$

Then $\bar{K}_{n}:[0, \pi] \times L^{2}([0, \pi]) \times L^{2}([0, \pi]) \rightarrow L_{Q}^{0}\left(L^{2}([0, \pi]), L^{2}([0, \pi])\right)$ be defined by

$$
\bar{K}_{n}(t, x, y) f(t)=\int_{0}^{t} K_{n}(t, s, x(s), y(s)) f(s) d s, \quad f \in L^{2}([0, \pi]),
$$


is a Hilbert-Schmidt operator and there exists $\alpha, \beta \geq 0$ such that $\sum_{n=1}^{\infty}\left\|\bar{K}_{n}(t, x, y)-\bar{K}_{n}(t, s, \bar{x}, \bar{y})\right\|_{L_{Q}^{0}}^{2} \leq \alpha\|x-\bar{x}\|_{L^{2}}^{2}+\beta\|y-\bar{y}\|_{L^{2}}^{2} \quad$ for each $x, \bar{x}, y, \bar{y} \in L^{2}([0, \pi])$.

Proof. To prove the result we use the same method in the proof of Corollary 7.2.

Lemma 7.1. Assume that $\sigma$ satisfies conditions of Corollary 7.1 or (Corollary 7.1 or 7.2), and (iii) or F, $G$ are Lipschitz functions and $I_{k}, I_{k}$ satisfy $(i)$ or $\left(i v^{\prime}\right)$. Then, problem 7.2 has at least one solution and problem (7.3) has exponentially stable mild solutions.

Proof. If we use that $F, G$ are Lipchitz functions and assumptions in Corollary 7.2 or 7.3 hold, then by Theorem 4.1, problem 7.2 possesses a unique mild solution. By the same method we can prove that all the conditions of Theorem 4.2 or 6.1 are satisfied.

Remark 7.1. In the case where $f_{2}=0, g^{1}=g^{2}=0, I_{k}=\bar{I}_{k}=0$ and $A=0$, our example includes the equation for Brownian motion on the group of diffeomorphisms of the circle (see [1,21]).

Acknowledgements. We would like to thank the anonymous referee and the editor for their careful reading of the manuscript and pertinent and helpful comments; their constructive suggestions allowed us to substantially improve the quality of our work. The research of T. Caraballo has been partially supported by FEDER and the Spanish Ministerio de Economía y Competitividad project MTM2015-63723-P and the Consejería de Innovación, Ciencia y Empresa (Junta de Andalucía) under grant 2010/FQM314 and Proyecto de Excelencia P12-FQM-1492.

\section{References}

[1] Hélène Airault and Jiagang Ren. Modulus of continuity of the canonic Brownian motion "on the group of diffeomorphisms of the circle". J. Funct. Anal., 196(2):395-426, 2002.

[2] M. Benchohra, J. Henderson, and S. Ntouyas. Impulsive differential equations and inclusions, volume 2 of Contemporary Mathematics and Its Applications. Hindawi Publishing Corporation, New York, 2006.

[3] A. T. Bharucha-Reid. Random integral equations. Academic Press, New YorkLondon, 1972. Mathematics in Science and Engineering, Vol. 96.

[4] Tayeb Blouhi, Juan J. Nieto, and Abdelghani Ouahab. Existence and uniqueness results for systems of impulsive stochastic differential equations. Ukrainian Math. J., (to appear). 
[5] Octavia Bolojan-Nica, Gennaro Infante, and Radu Precup. Existence results for systems with coupled nonlocal initial conditions. Nonlinear Anal., 94:231-242, 2014 .

[6] Ahmed Boudaoui, Tomás Caraballo, and Abdelghani Ouahab. Existence of mild solutions to stochastic delay evolution equations with a fractional Brownian motion and impulses. Stoch. Anal. Appl., 33(2):244-258, 2015.

[7] Guilan Cao and Kai He. On a type of stochastic differential equations driven by countably many Brownian motions. J. Funct. Anal., 203(1):262-285, 2003.

[8] Junfei Cao, Qigui Yang, Zaitang Huang, and Qing Liu. Asymptotically almost periodic solutions of stochastic functional differential equations. Appl. Math. Comput., 218(5):1499-1511, 2011.

[9] T. Caraballo, M. J. Garrido-Atienza, and T. Taniguchi. The existence and exponential behavior of solutions to stochastic delay evolution equations with a fractional Brownian motion. Nonlinear Anal., 74(11):3671-3684, 2011.

[10] Tomás Caraballo and Mamadou Abdoul Diop. Neutral stochastic delay partial functional integro-differential equations driven by a fractional Brownian motion. Front. Math. China, 8(4):745-760, 2013.

[11] Giuseppe Da Prato and Jerzy Zabczyk. Stochastic equations in infinite dimensions, volume 44 of Encyclopedia of Mathematics and its Applications. Cambridge University Press, Cambridge, 1992.

[12] Thomas C. Gard. Introduction to stochastic differential equations, volume 114 of Monographs and Textbooks in Pure and Applied Mathematics. Marcel Dekker, Inc., New York, 1988.

[13] Ц̆. I. Gīhman and A. V. Skorohod. Stochastic differential equations. SpringerVerlag, New York-Heidelberg, 1972. Translated from the Russian by Kenneth Wickwire, Ergebnisse der Mathematik und ihrer Grenzgebiete, Band 72.

[14] John R. Graef, Johnny Henderson, and Abdelghani Ouahab. Impulsive differential inclusions, volume 20 of De Gruyter Series in Nonlinear Analysis and Applications. De Gruyter, Berlin, 2013. A fixed point approach.

[15] A. Halanay and D. Wexler. Teoria calitativă a sistemelor cu impulsuri. Editura Academiei Republicii Socialiste România, Bucharest, 1968.

[16] Bjarne S. Jensen. The dynamic systems of basic economic growth models, volume 302 of Mathematics and its Applications. Kluwer Academic Publishers Group, Dordrecht, 1994. 
[17] V. Lakshmikantham, D. D. Bănov, and P. S. Simeonov. Theory of impulsive differential equations, volume 6 of Series in Modern Applied Mathematics. World Scientific Publishing Co., Inc., Teaneck, NJ, 1989.

[18] Chunxiang Li, Junping Shi, and Jitao Sun. Stability of impulsive stochastic differential delay systems and its application to impulsive stochastic neural networks. Nonlinear Anal., 74(10):3099-3111, 2011.

[19] Jun Liu, Xinzhi Liu, and Wei-Chau Xie. Existence and uniqueness results for impulsive hybrid stochastic delay systems. Comm. Appl. Nonlinear Anal., 17(3):3753, 2010.

[20] Meng Liu and Ke Wang. On a stochastic logistic equation with impulsive perturbations. Comput. Math. Appl., 63(5):871-886, 2012.

[21] Paul Malliavin. The canonic diffusion above the diffeomorphism group of the circle. C. R. Acad. Sci. Paris Sér. I Math., 329(4):325-329, 1999.

[22] Xuerong Mao. Stochastic differential equations and their applications. Horwood Publishing Series in Mathematics \& Applications. Horwood Publishing Limited, Chichester, 1997.

[23] V. D. Mil'man and A. D. Myškis. On the stability of motion in the presence of impulses. Sibirsk. Mat. Ž., 1:233-237, 1960.

[24] J. D. Murray. Mathematical biology, volume 19 of Biomathematics. SpringerVerlag, Berlin, 1989.

[25] David Nualart. The Malliavin calculus and related topics. Probability and its Applications (New York). Springer-Verlag, Berlin, second edition, 2006.

[26] Bernt Øksendal. Stochastic differential equations. Universitext. Springer-Verlag, Berlin, fourth edition, 1995. An introduction with applications.

[27] Lijun Pan and Jinde Cao. Exponential stability of impulsive stochastic functional differential equations. J. Math. Anal. Appl., 382(2):672-685, 2011.

[28] A. Pazy. Semigroups of linear operators and applications to partial differential equations, volume 44 of Applied Mathematical Sciences. Springer-Verlag, New York, 1983.

[29] A. I. Perov. On the Cauchy problem for a system of ordinary differential equations. Približ. Metod. Rešen. Differencial'. Uravnen. Vyp., 2:115-134, 1964.

[30] Radu Precup. Methods in nonlinear integral equations. Kluwer Academic Publishers, Dordrecht, 2002. 
[31] Radu Precup. The role of matrices that are convergent to zero in the study of semilinear operator systems. Math. Comput. Modelling, 49(3-4):703-708, 2009.

[32] Radu Precup and Adrian Viorel. Existence results for systems of nonlinear evolution equations. Int. J. Pure Appl. Math., 47(2):199-206, 2008.

[33] Ioan A. Rus. The theory of a metrical fixed point theorem: theoretical and applicative relevances. Fixed Point Theory, 9(2):541-559, 2008.

[34] R. Sakthivel and J. Luo. Asymptotic stability of nonlinear impulsive stochastic differential equations. Statist. Probab. Lett., 79(9):1219-1223, 2009.

[35] A. M. Samoŭlenko and N. A. Perestyuk. Impulsive differential equations, volume 14 of World Scientific Series on Nonlinear Science. Series A: Monographs and Treatises. World Scientific Publishing Co., Inc., River Edge, NJ, 1995. With a preface by Yu. A. Mitropol'skil and a supplement by S. I. Trofimchuk, Translated from the Russian by Y. Chapovsky.

[36] Kazimierz Sobczyk. Stochastic differential equations, volume 40 of Mathematics and its Applications (East European Series). Kluwer Academic Publishers Group, Dordrecht, 1991. With applications to physics and engineering.

[37] Chris P. Tsokos and W. J. Padgett. Random integral equations with applications to life sciences and engineering. Academic Press [A subsidiary of Harcourt Brace Jovanovich, Publishers], New York-London, 1974. Mathematics in Science and Engineering, Vol. 108.

[38] Shu-jin Wu, Xiao-lin Guo, and Song-qing Lin. Existence and uniqueness of solutions to random impulsive differential systems. Acta Math. Appl. Sin. Engl. Ser., 22(4):627-632, 2006.

[39] Quanxin Zhu and Bing Song. Exponential stability of impulsive nonlinear stochastic differential equations with mixed delays. Nonlinear Anal. Real World Appl., 12(5):2851-2860, 2011. 